\title{
CELL STRUCTURES ON THE BLOB ALGEBRA
}

\author{
STEEN RYOM-HANSEN
}

\begin{abstract}
We consider the $r=0$ case of the conjectures by Bonnafé, Geck, Iancu and Lam on cellular structures on the Hecke algebra of type $B$. We show that this case induces the natural cell structure on the blob algebra $b_{n}$ by restriction to one-line bipartitions.
\end{abstract}

\section{INTRODUCTION}

The purpose of this article is to continue the investigation, initiated in $\underline{\mathrm{RH}}$, of the relationship between the representation theories of the Hecke algebra $\mathcal{H}_{n}=$ $\mathcal{H}_{n}(Q, q)$ of type $B$ and of the blob algebra $b_{n}=b_{n}(q, m)$. The Hecke algebra $\mathcal{H}_{n}$ of type $B$ is a well-known two-parameter deformation of the hyperoctahedral group whereas the blob algebra $b_{n}$, introduced in [MS from motivations in statistical mechanics, is a diagram algebra of marked (blobbed) Temperley-Lieb diagrams. A main point of our work, already present in $[\mathrm{RH}]$, is that $b_{n}$ can also be realized as a quotient of $\mathcal{H}_{n}$ thus making the $b_{n}$-representations $\mathcal{H}_{n}$-representations by inflation. Viewing $b_{n}$ as a quotient of $\mathcal{H}_{n}$ is analogous to viewing the Temperley-Lieb algebra $T L_{n}$ as a quotient of the Hecke algebra of type $A$, and indeed $b_{n}$ is also sometimes called the Temperley-Lieb algebra of type $B$.

Dipper-James-Murphy introduced in DJM for each bipartition $(\lambda, \mu)$ of total degree $n$ a Specht module $S_{n}(\lambda, \mu)$ for $\mathcal{H}_{n}$. Let $J_{n}$ be the kernel of the quotient map $\mathcal{H}_{n} \rightarrow b_{n}$. We then showed in $\left[\mathrm{RH}\right.$ that $J_{n} S_{n}(\lambda, \mu)=0$ as long as $(\lambda, \mu)$ is a one-line bipartition and so these $S_{n}(\lambda, \mu)$ factor over the quotient map to become $b_{n}$-modules. One might now suspect that $S_{n}(\lambda, \mu)$ is a standard module for the quasi-hereditary algebra $b_{n}$. Indeed, we showed that many properties of the standard modules are shared by the $S_{n}(\lambda, \mu)$, but somewhat surprisingly we could prove in $[\mathrm{RH}$ that they do not verify the relevant universal property and so do not identify with standard modules, except in trivial cases.

Recall G. Lusztig's monograph [Lu2] on the representation theory of Hecke algebras with unequal parameters. Let $W$ be a Coxeter group and let $L: W \rightarrow \Gamma$ be a length function in the sense of [Lu2, for $\Gamma$ a totally ordered Abelian group. Associated to this data, Lu2 contains a construction of cells in $W$ and cell modules for the corresponding multiparameter Hecke algebra, generalizing the construction from $\overline{\mathrm{KL}}$ in the one-parameter case. When $W$ is of type $B$ the length function is specified by $a:=\log q, b:=\log Q \in \Gamma$. In BGIL a series of conjectures were formulated for type $B$ which, if true, would put a high degree of structure on this.

Received by the editors December 20, 2010 and, in revised form, March 7, 2011, March 1, 2012, and April 3, 2012.

2010 Mathematics Subject Classification. Primary 20G05, 20C08, 05E10.

This work was supported in part by FONDECYT grants 109070 and 1121129 , by Programa Reticulados y Simetría and by the MathAmSud project OPECSHA 01-math-10. 
Assume that $b \notin\{a, 2 a, \ldots,(n-1) a\}$ and that $a$ and $b$ are positive in $\Gamma$. According to the conjectures, the setting should give rise to a cellular algebra datum on $\mathcal{H}_{n}$ in the sense of Graham and Lehrer, where the underlying poset $\Lambda$ should be the set of bipartitions $\operatorname{Bip}(n)$ of total degree $n$ with partial order and map $\Lambda \times \Lambda \rightarrow \mathcal{H}_{n}$ defined in terms of a certain domino insertion algorithm, depending on $\Gamma$. Furthermore, by the work of Bonnafé and Jacon [BJ], the different cellular algebra structures on $\mathcal{H}_{n}$ should account for the different ways of parameterizing the simple modules for $\mathcal{H}_{n}$ that are given by Ariki's Theorem in $[\mathrm{A}$.

These conjectures have only been fully proved in the so-called asymptotic case $b>(n-1) a$ (see $[\mathrm{BI}])$ where the cell modules turn out to be the ones given by Dipper, James, and Murphy. In this work we focus on the case $\Gamma:=\mathbb{Z}, a:=2$ and $b=1$. This is another extreme case since $b<a$ and, so, $r=0$ in the notation used in BGIL. We show that the poset structure on $\operatorname{Bip}(n)$ in this case is compatible with the quasi-hereditary order on the category of $b_{n}$-modules when restricted to one-line bipartitions, the map being given by $(\lambda, \mu) \mapsto k-l$ where $\lambda=(k)$ and $\mu=(l)$. We show that the ideal $J_{n}$ is generated by the set of Kazhdan-Lusztig elements $C_{w}$ for which $w$ does not correspond to a one-line bipartition. Moreover, we show that the cell module given by the one-line bipartition $(\lambda, \mu)$ is isomorphic to the $b_{n}$ standard module $\Delta_{n}(k-l)$ where $\lambda=(k)$ and $\mu=(l)$. To summarize our findings: the $a=2, b=1$ case of the conjectures from BGIL] induces the blob algebra category when restricted to one-line bipartitions.

This given, the algorithm described in Ja can be used to answer the question that was raised in $[\mathrm{RH}$, namely to describe the Kleshchev bipartition that corresponds to the simple $b_{n}$-module $L_{n}(\lambda)$.

Let us indicate the layout of the article. The first section contains a combinatorial analysis of the domino insertion algorithm mentioned above. The main result is a characterization of the elements $W_{b}$ of the Weyl group $W_{n}$ of type $B$ that go to two-line tableaux under domino insertion. This characterization uses the Coxeter presentation of $W_{n}$. The section relies on results of Taskin, [T.

In the next section we recall the presentation of $b_{n}$ as a quotient of $\mathcal{H}_{n}$ and show that the defining ideal is given by the Kazhdan-Lusztig type elements $C_{w} \in \mathcal{H}_{n}$ where $w \notin W_{b}$. In the following section we show our main results, identifying the cell modules with the standard modules. To be more precise, we show that the cell modules verify the universal property for the standard modules, given within the framework of the globalization-localization formalism. For this to work we rely on Lusztig's results in [Lu1 that we combine with the results of Fan and Green [FG] on type $A$.

Finally, in the last section we show how the Fock space approach to the representation theory of $\mathcal{H}_{n}$ can be used to reprove the main results of [MW] and to obtain the Kleshchev bipartitions of the simple modules for $b_{n}$.

It is with great pleasure the author thanks the referees for many useful comments and suggestions.

\section{BASIC NOTATION AND DOMINO INSERTION}

In this section we first fix some notation that shall be used throughout the article. We then investigate the domino insertion algorithm for the Weyl group of type $B$. We describe the elements that are mapped under it to two-line partitions, that is domino tableaux whose underlying partition has at most two lines (parts). 
Throughout, we shall assume knowledge of the definition and basic properties of the Robinson-Schensted algorithm, as exposed for example in Sa].

For the following basic combinatorial concepts related to the Weyl group of type $B$, we refer the reader to section 8.1 of $\left[\mathrm{BB}\right.$. Let $W_{n}$ be the Weyl group of type $B_{n}$. It is a Coxeter group on generators $s_{0}, s_{1}, \ldots, s_{n-1}$ with relations

$$
\begin{array}{lr}
s_{i}^{2}=1 & \text { for } i=0, \ldots, n-1, \\
\left(s_{i} s_{i+1}\right)^{3}=1 & \text { for } i=1, \ldots, n-2, \\
\left(s_{i} s_{j}\right)^{2}=1 & \text { for }|i-j|>2, \\
\left(s_{0} s_{1}\right)^{4}=1 . &
\end{array}
$$

Let $I_{n}:=I_{n}^{+} \cup I_{n}^{-}$where $I_{n}^{+}:=\{1,2, \ldots, n\}$ and $I_{n}^{-}:=\{-1,-2, \ldots,-n\}$. Then $W_{n}$ can also be described as the subgroup of the symmetric group on the elements $I_{n}$ generated by $s_{0}:=(-1,1)$ and

$$
s_{i}:=(i, i+1)(-i,-i-1)
$$

in cycle notation. We shall adopt the convention that cycles are multiplied from right to left. The subgroup of $W_{n}$ generated by $s_{1}, s_{2}, \ldots, s_{n-1}$ is the symmetric group $S_{n}$.

For elements $w \in W_{n}$ we shall also use word or sequence notation such as

$$
w=i_{1} i_{2} i_{3} \ldots i_{n},
$$

where $i_{k} \in I_{n}$. By this we mean that $w$ acts (on the left) on $I_{n}$ as

$$
w: 1 \mapsto i_{1}, 2 \mapsto i_{2}, \ldots, n \mapsto i_{n}
$$

and then also necessarily $-1 \mapsto-i_{1},-2 \mapsto-i_{2}, \ldots,-n \mapsto-i_{n}$. In this setting we use the standard notation $\bar{i}:=-i \in I_{n}^{-}$for $i \in I_{n}^{+}$. Thus $i$ appears in $w=$ $i_{1} i_{2} i_{3} \ldots i_{n} \in W_{n}$ if and only if $\bar{i}$ does not not appear.

It is normally clear whether a given $w \in W_{n}$ is written as a product of Coxeter generators or as a word over $I_{n}$ and we shall therefore generally not explicitly mention the chosen form. If, for example, $w:=s_{0} s_{1} s_{2} \in W_{3}$, we may write

$$
w=s_{0} s_{1} s_{2}=23 \overline{1} \text {. }
$$

We denote by $<$ the Bruhat-Chevalley order on $W_{n}$ where by convention the neutral element $1 \in W_{n}$ is the smallest of all. Assume that $w=i_{1} i_{2} i_{3} \ldots i_{n} \in W_{n}$. Then the following conditions describe the right descent set of $w$ with respect to $<$ :

$$
\begin{aligned}
& w s_{k}<w \text { iff } i_{k}>i_{k+1} \quad \text { for } k=1,2, \ldots, n-1, \\
& w s_{0}<w \text { iff } i_{1}<0 .
\end{aligned}
$$

If $w \in W_{n}$ is written in word form, its right descent set can be used to write it as a reduced expression in the Coxeter generators $s_{i}$.

Example 2.1. Assume that $w=3 \overline{1} \overline{2} 4$. Then $s_{1} s_{0} s_{1} s_{0} s_{2} s_{1}$ is a reduced expression for $w$ obtained from the above description of the right descent set. Indeed, $s_{2} s_{1}$ moves 3 past $\overline{1} \overline{2}$, then $s_{0}$ changes $\overline{1}$ to 1 and finally $s_{1} s_{0} s_{1}$ changes $\overline{2}$ to 2 .

Throughout the paper, we shall be especially interested in the subset $W_{b}=$ $W_{n, b}$ of $W_{n}$. It consists of those $w \in W_{n}$ that have no reduced expressions $w=$ $s_{i_{1}} s_{i_{2}} s_{i_{3}} \ldots s_{i_{N}}$ that contain a subexpression $s_{i_{k}} s_{i_{k+1}} s_{i_{k+2}}$ of the form

$$
s_{i} s_{i \pm 1} s_{i} \text { for } i=1,2, \ldots, n-2 \text { or } s_{n-1} s_{n-2} s_{n-1} .
$$

Thus the subexpression $s_{0} s_{1} s_{0}$ is allowed whereas $s_{1} s_{0} s_{1}$ is not. 
Our aim is to describe the image of $W_{b}$ under the domino insertion correspondence described, for example, in BGIL. In order to do so we first need a description of $W_{b}$ in terms of words. This description will only be indirect, but for our purposes this will be sufficient.

In general we use the convention that empty index sequences correspond to the void subsequence. For example, in the next lemma, the case $k=1$ corresponds to $w=\bar{a}_{1} i_{2} \ldots i_{n}$.

Lemma 2.2. Assume that $w \in W_{n}$ and assume that it can be written as

$$
w=i_{1} i_{2} \ldots i_{k-1} \bar{a}_{1} i_{k+1} \ldots i_{n},
$$

where $i_{1}, i_{2}, \ldots i_{k-1}, a_{1}>0$. Then $w \in W_{b}$ if and only if

$$
a_{1}<i_{1}<i_{2}<\ldots<i_{k-1} \quad \text { and } a_{1} i_{1} i_{2} \ldots i_{k-1} i_{k+1} \ldots i_{n} \in W_{b} .
$$

Proof. Suppose that $w \in W_{b}$ and define $w_{1} \in W_{n}$ as

$$
w_{1}=a_{1} i_{1} i_{2} \ldots i_{k-1} i_{k+1} \ldots i_{n} .
$$

Using the above description of the right descent set we get that $w$ has a reduced expression of the form

$$
w=w_{1} s_{0} s_{1} \ldots s_{k-2} s_{k-1}
$$

and the second statement follows, since any reduced expression for $w_{1}$ can be extended to a reduced expression for $w$.

Now, if $a_{1}<i_{1}<i_{2}<\ldots<i_{k-1}$ is not satisfied, then by the description of the right descent set there will be an index $1 \leq j \leq k-1$ such that $w_{1} s_{j}<w_{1}$. But by formula $(*)$ this contradicts the assumption that $w \in W_{b}$.

To show the other implication we assume that $a_{1}<i_{1}<\ldots<i_{k-1}$ holds, that $w_{1}=a_{1} i_{1} i_{2} \ldots i_{k-1} i_{k+1} \ldots i_{n} \in W_{b}$ and that $w \notin W_{b}$. Since $s_{0} s_{1} s_{2} \ldots s_{k-1}$ is a unique representation for $w_{1}^{-1} w$ and since $w_{1} \in W_{b}$ we conclude that $w_{1}$ must have a reduced expression of the form $w_{1}:=w_{2} s_{j}$, for an index $j$ such that $0 \leq j \leq k-1$. But then $s_{j}$ belongs to the right descent set for $w_{1}$, contradiction.

Before stating our next result, we need to recall the combinatorial notion of a decreasing subsequence. Suppose that $w=i_{1} i_{2} i_{3} \ldots i_{n} \in W_{n}$. A decreasing subsequence of $w$ of length $k$ is defined to be a subsequence $i_{\iota_{1}} i_{\iota_{2}} \ldots i_{\iota_{k}}$ of $w$ such that $\iota_{j}<\iota_{j+1}$ and $i_{\iota_{j}}>i_{\iota_{j+1}}$ for $j=1, \ldots, k$.

Setting $W_{c}:=W_{b} \cap S_{n}$, it is known that $W_{c}$ can be described as the words over $I_{n}^{+}$with no decreasing subsequences of length strictly greater than two and so it corresponds under the Robinson-Schensted algorithm to pairs of tableaux $(s, t)$ of a two-line partition $\lambda=\left(\lambda_{1}, \lambda_{2}\right)$.

Theorem 2.3. Suppose $w \in W_{n}$ and write it as

$$
w=i_{1} i_{2} \ldots i_{k_{1}-1} \bar{a}_{1} i_{k_{1}+1} \ldots i_{k_{2}-1} \bar{a}_{2} i_{k_{2}+1} \ldots i_{k_{l}-1} \bar{a}_{l} i_{k_{l}+1} \ldots i_{n}
$$

where $\bar{a}_{1}, \ldots, \bar{a}_{l}$ are the only negative numbers that occur in $w$. Define

$$
w^{l}:=a_{l} a_{l-1} \ldots a_{1} i_{1} i_{2} \ldots i_{n} .
$$

Then $w \in W_{b}$ if and only if

$$
a_{l}<a_{l-1}<\ldots<a_{1}<i_{1}<i_{2}<\ldots<i_{k_{l}-1}
$$

and $w^{l}$ has no decreasing subsequences of length strictly greater than two. 


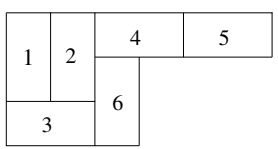

Figure 1

Proof. Suppose first that $w \in W_{b}$. We generalize $w^{l}$ as follows:

$$
\begin{aligned}
& w^{1}=a_{1} i_{1} i_{2} \ldots i_{k_{1}-1} i_{k_{1}+1} \ldots i_{k_{2}-1} \bar{a}_{2} i_{k_{2}+1} \ldots i_{k_{l}-1} \bar{a}_{l} i_{k_{l}+1} \ldots i_{n}, \\
& w^{2}=a_{2} a_{1} i_{1} i_{2} \ldots i_{k_{1}-1} i_{k_{1}+1} \ldots i_{k_{2}-1} i_{k_{2}+1} \ldots i_{k_{l}-1} \bar{a}_{l} i_{k_{l}+1} \ldots i_{n}, \\
& \vdots \\
& w^{l}=a_{l} a_{l-1} \ldots a_{1} i_{1} i_{2} \ldots i_{k_{1}-1} i_{k_{1}+1} \ldots i_{k_{2}-1} i_{k_{2}+1} \ldots i_{k_{l}-1} i_{k_{l}+1} \ldots i_{n} .
\end{aligned}
$$

By the proof of the previous lemma we have $w^{k} \in W_{b}$ for all $k$ and so we get the inequalities

$$
a_{l}<a_{l-1}<\ldots<a_{1}<i_{1}<i_{2}<\ldots<i_{k_{l}-1}
$$

by using the previous lemma recursively. But $w^{l} \in W_{b} \cap S_{n}$ and so we have proved one implication of the theorem.

The other implication follows in a similar way from the previous lemma.

Example 2.4. Let us consider $w=3 \overline{1} \overline{2} 4$ from the previous example. Then, in the notation of the theorem, we have $w^{2}:=2134$ and so $w \notin W_{b}$, since 213 is not increasing. The conclusion could also have been obtained directly from the definition of $W_{b}$ and the description of $w$ found in the previous example: $w=$ $s_{1} s_{0} s_{1} s_{0} s_{2} s_{1}$.

Example 2.5. Using the theorem, one can produce elements of $W_{b}$ by shuffling an increasing sequence of negative numbers with an increasing sequence of positive numbers, such that the positive terms all have absolute values larger than the negative numbers. For example,

$$
w:=\overline{4} \overline{3} 5 \overline{2} 678 \overline{1} 9 \in W_{9, b} .
$$

The notion of domino tableaux shall be important to us. A domino tableau is the Young diagram of an integer partition of $2 n$ with node set partioned into dominoes, that is horizontally or vertically neighboring nodes. The dominoes are labeled with numbers $1,2, \ldots, n$. A domino tableau is called standard if the labeling is increasing from left to the right and from top to bottom. Let $S D T(n)$ denote the set of standard domino tableaux in $n$ dominoes. Figure 1 gives an example from $S D T(6)$. We define $S D T:=\bigcup_{n} S D T(n)$. For $S \in S T D$ we let $S h(S)$ denote the shape of its underlying partition. Let $S D T^{2}(n)$ be the set

$$
S D T^{2}(n):=\{(S, T) \in S D T(n) \times S D T(n) \mid S h(S)=S h(T)\} .
$$

The domino insertion algorithm establishes a bijection between $W_{n}$ and $S D T^{2}(n)$. It was introduced in [BV] as a generalization of the Robinson-Schensted algorithm to type $B$. A slightly different version of the algorithm, using a bumping procedure, was introduced in $\mathrm{Ga}$; see also $\mathrm{vL}$. We shall not give here a precise description of the algorithm, but refer the reader, for instance, to [BGIL] or [La].

Let us denote by $(P(w), Q(w))$ the pair of domino tableaux associated with $w \in W_{n}$ under domino insertion. We say that $w$ and $w_{1}$ belong to the same Knuth 
(plactic) class, or $w \stackrel{p}{\sim} w_{1}$, if $P(w)=P\left(w_{1}\right)$. Dually, we say that $w$ and $w_{1}$ belong to the same dual Knuth (coplactic) class, or $w \stackrel{p^{*}}{\sim} w_{1}$, if $Q(w)=Q\left(w_{1}\right)$.

Taskin considers in Definition 3.1 of [T] a set of generalizations of the Knuth relations, $D_{i}^{r}, i=1,2, \ldots 5$, that generate the (co)plactic relations. We now explain these relations in the case that we need, namely $r=0$, where they simplify somewhat. The elements of $W_{n}$ are always assumed to be in word form.

Let $f: I_{n} \rightarrow I_{n}$ be any bijection such that $f(1)<f(2)<f(3)$. Then $D_{1}^{0}$ can be reformulated as the combination of the two relations

$$
\begin{aligned}
& \cdots f(2) f(3) f(1) \cdots \stackrel{K T}{\sim} \cdots f(2) f(1) f(3) \cdots, \\
& \cdots f(1) f(3) f(2) \cdots \stackrel{K T}{\sim} \cdots f(3) f(1) f(2) \cdots,
\end{aligned}
$$

where we use the convention that there are no changes of dotted elements. The relation $D_{2}^{0}$ is void whereas the relation $D_{3}^{0}$ is

$$
i_{1} i_{2} \ldots \stackrel{K T}{\sim} \overline{i_{1}} i_{2} \ldots \quad \text { if }\left|i_{1}\right|>\left|i_{2}\right|
$$

under a further condition on the dotted elements that we do not need to detail.

The remaining two relations $D_{4}^{0}$ and $D_{5}^{0}$ are more complicated to express than the first ones. But since we are only considering the $r=0$ case of Taskin's results, we may use a somewhat simplified notation.

Let us first consider $D_{4}^{0}$. Assume that $\alpha$ and $\alpha^{\prime}$ are elements of $W_{n}$ that can be expressed as $\alpha=u \ldots$ and $\alpha^{\prime}=u^{\prime} \ldots$ where

$$
\begin{gathered}
u=a_{1,1} b_{1,1} a_{2,2} a_{2,1} b_{2,2} b_{2,1} \ldots a_{k, k} a_{k, k-1} \ldots \\
a_{k, 1}\left(b_{k, k} b_{k, k-1} \ldots b_{k, 1}\right) a_{k+1, k} \ldots a_{k+1,1} z, \\
u^{\prime}=a_{1,1} b_{1,1} a_{2,2} a_{2,1} b_{2,2} b_{2,1} \ldots\left(-b_{k, k}\right) a_{k, k} a_{k, k-1} \ldots \\
a_{k, 1}\left(b_{k, k-1} \ldots b_{k, 1}\right) a_{k+1, k} \ldots a_{k+1,1} z
\end{gathered}
$$

for some $z \in I_{n}$ and $k \geq 1$ (notice that there is no $a_{k+1, k+1}$ ). Suppose, moreover, that $\left\{a_{i, j}\right\}_{i, j \geq 1}$ and $\left\{b_{i, j}\right\}_{i, j \geq 1}$ satisfy

$$
\begin{aligned}
& a_{i, j}>0 \text { and } b_{i, j}<0 \text { (or vice versa) for all } i, j \geq 1, \\
& \left|a_{i, j-1}\right|<\left|a_{i, j}\right|<\left|a_{i+1, j}\right| \text { and }\left|b_{i, j-1}\right|<\left|b_{i, j}\right|<\left|b_{i+1, j}\right|, \\
& \left|b_{i, i}\right|<\left|a_{i+1, i+1}\right|<\left|b_{i+1, i+1}\right| \text { for all } i=1, \ldots, k-1
\end{aligned}
$$

Then $D_{4}^{0}$ is the relation

$$
\alpha \stackrel{K T}{\sim} \alpha^{\prime}
$$

under certain further conditions on $z$ that we do not detail.

Let us finally consider the relation $D_{5}^{0}$. Assume that $\alpha$ and $\alpha^{\prime}$ are elements of $W_{n}$ that can be expressed as $\alpha=u \ldots$ and $\alpha^{\prime}=u^{\prime} \ldots$ where

$$
\begin{array}{r}
u=a_{1,1} b_{1,1} \ldots\left(a_{k, k} \ldots a_{k, 1}\right)\left(b_{k, k} \ldots b_{k, 1}\right)\left(a_{k+1, k+1} a_{k+1, k} \ldots a_{k+1,1}\right)\left(b_{k+1, k} \ldots b_{k, 1}\right) z \\
u^{\prime}=a_{1,1} b_{1,1} \ldots\left(a_{k, k} \ldots a_{k, 1}\right)\left(-a_{k+1, k+1}\right)\left(b_{k, k} \ldots b_{k, 1}\right) \\
\left(a_{k+1, k} \ldots a_{k+1,1}\right)\left(b_{k+1, k} \ldots b_{k, 1}\right) z
\end{array}
$$


for $z \in I_{n}$ and $k \geq 1$ (this time there is no $b_{k+1, k+1}$ ). Assume, moreover, that $\left\{a_{i, j}\right\}_{i, j \geq 1}$ and $\left\{b_{i, j}\right\}_{i, j \geq 1}$ satisfy

$$
\begin{aligned}
& a_{i, j}>0 \text { and } b_{i, j}<0 \text { (or vice versa) for all } i, j \geq 1, \\
& \left|a_{i, j-1}\right|<\left|a_{i, j}\right|<\left|a_{i+1, j}\right| \text { and }\left|b_{i, j-1}\right|<\left|b_{i, j}\right|<\left|b_{i+1, j}\right|, \\
& \left|a_{i, i}\right|<\left|b_{i, i}\right|<\left|a_{i+1, i+1}\right| \text { for all } i=1, \ldots, k
\end{aligned}
$$

Then $D_{5}^{0}$ is the relation that

$$
\alpha \stackrel{K T}{\sim} \alpha^{\prime}
$$

under certain further conditions on $z$ that, once again, we do not detail.

We shall refer to the relations (2.1), (2.2), (2.3), (2.4) and (2.5) as the KnuthTaskin relations. Note that they are read either from the left to the right or conversely. The main results, Theorem 3.4 and Theorem 3.5 of [T], amount in the $r=0$ case to the following:

Theorem 2.6. Suppose $w, z \in W_{n}$. Then they belong to the same plactic class if and only if there is a sequence $w_{1}, w_{2}, \ldots, w_{k} \in W_{n}$ such that $w=w_{1}, z=w_{k}$ and $w_{i} \stackrel{K T}{\sim} w_{i+1}$ for $i=1,2, \ldots, k-1$. In other words, the plactic classes are generated by the Knuth-Taskin relations.

The dual Knuth-Taskin relations are defined by $w \stackrel{D K T}{\sim} w_{1}$ if $w^{-1} \stackrel{K T}{\sim} w_{1}^{-1}$. If $w$ and $w_{1}$ are written in word form, they do not act on neighboring elements, and as a matter of fact, they do not admit as a simple description as in the symmetric group case. On the other hand, since $Q(w)=P\left(w^{-1}\right)$, we get an obvious dual version of the previous theorem:

Theorem 2.7. Suppose $w, z \in W_{n}$. Then they belong to the same coplactic class if and only if there is a sequence $w_{1}, w_{2}, \ldots, w_{k} \in W_{n}$ such that $w=w_{1}, z=w_{k}$ and $w_{i} \stackrel{D K T}{\sim} w_{i+1}$ for $i=1,2 \ldots, k-1$.

We need the following lemma.

Lemma 2.8. $W_{b}$ is stable under the Knuth-Taskin relations (2.1), (2.2), (2.3), (2.4) and (2.5).

Proof. Assume that $w \in W_{b}$ and write it in the form

$$
w=\underline{i}_{1} \bar{a}_{1} \underline{i}_{2} \bar{a}_{2} \cdots \underline{i}_{l} \bar{a}_{l} w_{1}
$$

where $w_{1}, \underline{i}_{j}$ are words, possibly empty, over $I_{n}^{+}$for $j=1,2, \ldots, l$ and $a_{j}>0$ for $j=1,2, \ldots, l$. Note that we allow $l=0$ which corresponds to $w=w_{1}$. Write

$$
\underline{i}_{1} \underline{i}_{2} \ldots \underline{i}_{l} w_{1}=i_{1} i_{2} \ldots i_{k} .
$$

Assume now that the Knuth-Taskin relation (2.1) acts in the

$$
\underline{i}_{1} \bar{a}_{1} \underline{i}_{2} \bar{a}_{2} \ldots \underline{i}_{l} \bar{a}_{l}
$$

part of $w$. We know from Theorem 2.3 that all $\underline{i}_{j}$ are increasing sequences over $I_{n}^{+}$ and that

$$
a_{l}<a_{l-1}<a_{l-2}<\ldots<a_{1}<\underline{i}_{1}<\underline{i}_{2}<\ldots<\underline{i}_{l}
$$

where the inequalities hold for all elements of the subsequences, and so the pattern $f(2) f(3) f(1)$ can only occur if $f(1)=\bar{a}_{r}$ for some $1 \leq r \leq l$ and $f(3)=i_{s}$ for some $s$. But then clearly (2.1) takes $w$ to another element of $W_{b}$. Likewise, we 
see that (2.1) acting in the pattern $f(2) f(1) f(3)$ of $\underline{i}_{1} \bar{a}_{1} \underline{i}_{2} \bar{a}_{2} \ldots \underline{i}_{l} \bar{a}_{l}$ takes $w$ to another element of $W_{b}$.

In the case of the Knuth-Taskin relation (2.2) acting in

$$
\underline{i}_{1} \bar{a}_{1} \underline{i}_{2} \bar{a}_{2} \ldots \underline{i}_{l} \bar{a}_{l}
$$

we argue similarly. By the inequalities (2.6), the only decreasing subsequences of $\underline{i}_{1} \bar{a}_{1} \underline{i}_{2} \bar{a}_{2} \ldots \underline{i}_{l} \bar{a}_{l}$ are of the form $i_{r} \bar{a}_{s}$ for some $r, s$ and so in the pattern $f(1) f(3) f(2)$ we have that $f(3)=i_{r}$ for some $r$ whereas $f(2)=\bar{a}_{s}$ for some $s$. But since $f(1)$ is less than $f(2)$ it must be $\bar{a}_{t}$ for some $t$ and so changing $f(1) f(3) f(2)$ to $f(3) f(1) f(2)$ gives another element of $W_{b}$. We argue similarly in the case of the pattern $f(3) f(1) f(2)$.

Assume now that one of the Knuth-Taskin relation (2.1) or (2.2) acts in the $w_{1}$ part of $w$. By the theory of the usual Robinson-Schensted algorithm, the length of the longest decreasing subsequence is preserved when the action is on words over $I_{n}^{+}$, and hence we get from Theorem 2.3 that (2.1) and (2.2) map $w$ to an element of $W_{b}$ in this case.

We then consider the case where the action of one of the Knuth-Taskin relations (2.1) and (2.2) involves both $\underline{i}_{1} \bar{a}_{1} \underline{i}_{2} \bar{a}_{2} \ldots \underline{i}_{l} \bar{a}_{l}$ and $w_{1}$. In that case $\bar{a}_{l}$ must occur in the first or second position of the relation.

Case $f(2) f(3) f(1)$ : This case does not occur since $f(1)$ would belong to $w_{1}$ and would be less than $\bar{a}_{l}$, which contradicts the fact that $w_{1}$ is a word over $I_{n}^{+}$.

Case $f(2) f(1) f(3)$ : Using once again that the only decreasing subsequences of $\underline{i}_{1} \bar{a}_{1} \underline{i}_{2} \bar{a}_{2} \ldots \underline{i}_{l} \bar{a}_{l}$ are of the form $i_{r} \bar{a}_{s}$, we get in this case that $f(1)=\bar{a}_{l}$ whereas $f(2)$ is unbarred. Applying the Knuth-Taskin relation (2.1) yields $f(2) f(3) f(1)$, and hence $\underline{i}_{l}$ changes to $\underline{i}_{l} f(3)$, which is still increasing.

Case $f(1) f(3) f(2)$ : In this case we have that $f(1)=\bar{a}_{l}$ and $f(3)$ and $f(2)$ are unbarred, since $f(2) \in w_{1}$ and $f(3)>f(2)$. Thus also $f(3) \in w_{1}$. The application of the Knuth-Taskin relation (2.2) changes $f(1) f(3) f(2)$ to $f(3) f(1) f(2)$ and hence $\underline{i}_{l}$ changes to $\underline{i}_{l} f(3)$. But no element of $\underline{i}_{l}$ can be larger than $f(3)$ for if $i_{r}$ were such an element, then we may assume it is the last one of $\underline{i}_{l}$ and $i_{r} f(3) f(2)$ would be a decreasing subsequence longer than three, inside $a_{l} a_{l-1} a_{l-2} \ldots a_{1} \underline{i}_{1} \underline{i}_{2} \ldots \underline{i}_{l} w_{1}$. Thus $\underline{i}_{l} f(3)$ is increasing and we are done in this case as well.

Case $f(3) f(1) f(2)$ : We have $f(1)=\bar{a}_{l}$. Using the Knuth-Taskin relation (2.2) we have that $f(3) f(1) f(2)$ changes to $f(1) f(3) f(2)$ and thus $\underline{i}_{l}$ changes to $\underline{i}_{l} \backslash f(3)$ which is clearly increasing.

We next check that also the third Knuth-Taskin relation (2.3) takes $w \in W_{b}$ to an element of $W_{b}$. If $w=w_{1}$, then $i_{1}>i_{2}$ since we are supposing that (2.3) acts in $w$. Using Theorem 2.3 we then find that the image of $w$ under (2.3), namely $\bar{i}_{1} i_{2} \ldots$ also belongs to $W_{b}$.

In the remaining cases at least one of the two first elements of $w$ must be negative. Using Theorem 2.3 they are either of the form $i_{1} \overline{a_{2}}$ with $i_{1}>a_{2}$ or $\bar{a}_{1} \bar{a}_{2}$ with $a_{1}>a_{2}$. But then from Theorem 2.3 once again we find in each case that the image of $w$ under (2.3) also belongs to $W_{b}$.

We finally show that the Knuth-Taskin relations (2.4) and (2.5) map $w$ to an element of $W_{b}$. For this we assume that $w \in W_{b}$ is either of the form $w=\alpha$ or $w=\alpha^{\prime}$ in the description of (2.4) and (2.5), and we let $u, u^{\prime}$ and $k$ be chosen correspondingly. 
Let us first consider the Knuth-Taskin relation (2.4). We claim that if $k \geq 2$ and either $\alpha$ or $\alpha^{\prime}$ belongs to $W_{b}$, then the Knuth-Taskin relation (2.4) does not apply. Let us first verify this for $k \geq 3$. In that case $u$ and $u^{\prime}$ are both of the form

$$
a_{1,1} b_{1,1} a_{2,2} a_{2,1} b_{2,2} b_{2,1} \cdots
$$

where the dotted elements contain both $a_{3,1}$ and $b_{3,1}$ and hence both positive and negative numbers. By the conditions on (2.4) we have the inequalities $\left|b_{2,1}\right|<\left|b_{2,2}\right|$, $\left|a_{2,1}\right|<\left|a_{2,2}\right|$ and $\left|b_{1,1}\right|<\left|a_{2,2}\right|<\left|b_{2,2}\right|$, among others. If $a_{i, j}>0$ (or equivalently $b_{i, j}<0$ ) we get a contradiction with Theorem 2.3 that implies $\left|a_{2,2}\right|<\left|a_{2,1}\right|$ if $\alpha$ or $\alpha^{\prime}$ belongs to $W_{b}$. If $a_{i, j}<0$ (or $b_{i, j}>0$ ) we also get a contradiction with Theorem 2.3 that implies $\left|b_{2,2}\right|<\left|b_{2,1}\right|$, and the claim is proved for $k \geq 3$.

In the case $k=2$ we have

$$
u=a_{1,1} b_{1,1} a_{2,2} a_{2,1} b_{2,2} b_{2,1} a_{3,2} a_{3,1} z \text { and } u^{\prime}=a_{1,1} b_{1,1}\left(-b_{2,2}\right) a_{2,2} a_{2,1} b_{2,1} a_{3,2} a_{3,1} z
$$

and the same inequalities hold, that is, $\left|b_{2,1}\right|<\left|b_{2,2}\right|,\left|a_{2,1}\right|<\left|a_{2,2}\right|$ and $\left|b_{1,1}\right|<$ $\left|a_{2,2}\right|<\left|b_{2,2}\right|$. We can then argue as above to show that $\alpha$ cannot belong to $W_{b}$, indeed, $b_{i, j}<0$ implies by Theorem 2.3 that $\left|a_{2,2}\right|<\left|a_{2,1}\right|$ and $b_{i, j}>0$ implies $\left|b_{2,2}\right|<\left|b_{2,1}\right|$. Similarly, if $\alpha^{\prime} \in W_{b}$ and $b_{i, j}<0$ we get from Theorem 2.3 that $\left|a_{2,2}\right|<\left|a_{2,1}\right|$, which is a contradiction and if $b_{i, j}>0$ we get from Theorem 2.3 that $\left|b_{2,2}\right|<\left|a_{1,1}\right|$, which is also a contradiction.

The only remaining possibility is $k=1$. In that case we have

$$
u=a_{1,1} b_{1,1} a_{2,1} z, \quad u^{\prime}=\left(-b_{1,1}\right) a_{1,1} a_{2,1} z
$$

where $\left|a_{1,1}\right|<\left|a_{2,1}\right|$. If $b_{1,1}<0$ we get by Theorem 2.3 that $\alpha \in W_{b}$ iff $\alpha^{\prime} \in W_{b}$. On the other hand, if $b_{1,1}>0$ we have that $a_{1,1}$ and $a_{2,1}$ are negative and hence by the inequality neither $\alpha$ nor $\alpha^{\prime}$ belongs to $W_{b}$, using Theorem 2.3 once again.

We then finally treat the Knuth-Taskin relation (2.5). We proceed in the same way as for (2.4). If $k \geq 3$ we have that $u$ and $u^{\prime}$ are both of the form (2.7) where the dotted elements contain both positive and negative numbers and where $\left|b_{2,1}\right|<\left|b_{2,2}\right|,\left|a_{2,1}\right|<\left|a_{2,2}\right|$ and $\left|a_{1,1}\right|<\left|b_{1,1}\right|<\left|a_{2,2}\right|$, among others. The first two inequalities are the same as for (2.4) and so the argument used for (2.4) shows that neither $\alpha$ nor $\alpha^{\prime}$ can belong to $W_{b}$.

When $k=2$, we also use essentially the same argument as for (2.4). Indeed, we have

$$
\begin{aligned}
& u=a_{1,1} b_{1,1} a_{2,2} a_{2,1} b_{2,2} b_{2,1} a_{3,3} a_{3,2} a_{3,1} b_{3,2} b_{3,1} z, \\
& u^{\prime}=a_{1,1} b_{1,1} a_{2,2} a_{2,1}\left(-a_{3,3}\right) b_{2,2} b_{2,1} a_{3,2} a_{3,1} b_{3,2} b_{3,1} z
\end{aligned}
$$

and the same inequalities hold, that is, $\left|b_{2,1}\right|<\left|b_{2,2}\right|,\left|a_{2,1}\right|<\left|a_{2,2}\right|$ and $\left|a_{1,1}\right|<$ $\left|b_{1,1}\right|<\left|a_{2,2}\right|$. These inequalities ensure, using Theorem 2.3 as before, that $\alpha$ does not belong to $W_{b}$. On the other hand, if $b_{i, j}<0$ and $\alpha^{\prime} \in W_{b}$, we get by Theorem 2.3 that $\left|a_{2,2}\right|<\left|a_{2,1}\right|$, which is a contradiction. Finally, if $b_{i, j}>0$ and $\alpha^{\prime} \in W_{b}$ we get that $\left|a_{2,2}\right|<\left|b_{1,1}\right|$, which is also a contradiction.

The only remaining case is now $k=1$ where we have

$$
u=a_{1,1} b_{1,1} a_{2,2} a_{2,1} b_{2,1} z, \quad u^{\prime}=a_{1,1}\left(-a_{2,2}\right) b_{1,1} a_{2,1} b_{2,1} z,
$$

where $\left|a_{2,1}\right|<\left|a_{2,2}\right|$ and $\left|a_{1,1}\right|<\left|b_{1,1}\right|<\left|a_{2,2}\right|$. These inequalities imply by Theorem 2.3 that neither $\alpha$ nor $\alpha^{\prime}$ is in $W_{b}$. The lemma is proved.

Corollary 2.9. $W_{b}$ is a union of plactic classes and also a union of coplactic classes. 


\begin{tabular}{|l|l|l|l|l|l|l|}
\hline \multirow{2}{*}{1} & 2 &.. & $\mathrm{k}-1$ & $\mathrm{k}+1$ & & $\mathrm{n}$ \\
\cline { 2 - 7 } & 3 &.. & $\mathrm{k}$ & \multicolumn{3}{|c}{} \\
\hline
\end{tabular}

\begin{tabular}{|c|c|c|c|c|c|c|}
\hline 1 & 3 &.. & $\mathrm{k}-1$ & & .. & $\mathrm{n}$ \\
\hline 2 & 4 &.. & $\mathrm{k}$ & \multicolumn{3}{|c}{} \\
\cline { 1 - 3 }
\end{tabular}

FIGURE 2

\begin{tabular}{|l|l|l|l|l|l|l|l|l|l|l|}
\hline $\mathrm{a}_{1}$ & $\mathrm{a}_{2}$ &.. & $\mathrm{a}_{\mathrm{u}}$ & $\mathrm{i}_{1}$ &.. & $\mathrm{i}_{\mathrm{t}}$ & $\mathrm{i}_{\mathrm{t}+1}$ &.. & & $\mathrm{i}_{\mathrm{k}}$ \\
\cline { 5 - 8 } & & & & & &
\end{tabular}

FigURE 3

Proof. The previous lemma amounts to saying that $W_{b}$ is a union of plactic classes. But $Q(w)=P\left(w^{-1}\right)$ and $W_{b}$ is stable with respect to $w \mapsto w^{-1}$, hence $W_{b}$ is also a union of coplactic classes.

For $w \in W_{n}$ we define $S h(w)$ by $S h(P(w))$ or, equivalently, by $S h(Q(w))$. Define $S T D_{\leq 2}^{2}:=\left\{(S, T) \in S T D^{2} \mid S h(S)\right.$ has less than two lines $\}$.

We are now in position to prove the main theorem of this section.

Theorem 2.10. Suppose that $w \in W_{n}$. Then $w \in W_{b}$ if and only if $S h(w)$ is a Young diagram of at most two lines. In other words, $W_{b}$ is in correspondence with $S T D_{\leq 2}^{2}$ under domino insertion.

Proof. Assume first that $S h(w)$ has at most two lines. Using Theorems 2.6 and 2.7 there is $w_{1} \in W_{n}$ related to $w$ through a series of Knuth-Taskin or dual KnuthTaskin relations such that $P\left(w_{1}\right)$ and $Q\left(w_{1}\right)$ both have one of the forms given in Figure 2 depending on the parity of the first line of $S h(w)$. Under the domino insertion algorithm, the first tableau corresponds to

$$
13254 \ldots k k-1 k+1 k+2 k+3 \ldots n
$$

whereas the second tableau corresponds to

$$
214365 \ldots k k-1 k+1 k+2 k+3 \ldots n .
$$

Since they both belong to $W_{b}$ we deduce from Lemma 2.8 that $w$ also belongs to $W_{b}$ and one implication of the theorem is proved.

To prove the other implication we take $w \in W_{b}$ and show that $P(w)$ has at most two lines. First, write $w$ in the form

$$
w=\underline{i}_{1} \bar{a}_{1} \underline{i}_{2} \bar{a}_{2} \ldots \underline{i}_{u} \bar{a}_{u} w_{1}
$$

where $w_{1}, \underline{i}_{j}$ are words over $I_{n}^{+}$and $a_{j}>0$. We set

$$
i_{1} i_{2} i_{3} \ldots i_{k}:=\underline{i}_{1} \underline{i}_{2} \ldots \underline{i}_{u} .
$$

By Theorem 2.3 there is now a $t$ such that $P:=P\left(\underline{i}_{1} \bar{a}_{1} \underline{i}_{2} \bar{a}_{2} \ldots \underline{i}_{u} \bar{a}_{u}\right)$ is the domino tableau given in Figure 3. Let $w_{1}=j_{1} j_{2} \ldots j_{n-k-u}$ and let $j_{i_{1}} j_{i_{2}} \ldots j_{i_{r}}$ be the subsequence of $w_{1}$ consisting of those elements $j_{i}$ that are less then $i_{k}$. Then by Theorem 2.3 we have that $j_{i_{1}} j_{i_{2}} \ldots j_{i_{r}}$ is an increasing subsequence. Let $j_{\iota_{1}} j_{\iota_{2}} \ldots j_{\iota_{s}}$ be the subsequence of $w_{1}$ consisting of those elements that are positioned before $j_{i_{r}}$ in $w_{1}$ and are bigger than $i_{k}$. By Theorem 2.3 this is also an increasing subsequence. Setting $z_{1}:=j_{1} j_{2} \ldots j_{i_{r}}$ and $z_{2}:=j_{i_{r}+1} j_{i_{r}+2} \ldots j_{n-k-u}$ we obviously have that $w_{1}=z_{1} z_{2}$. Moreover, $z_{1}$ is a shuffle of its subsequences $j_{i_{1}} j_{i_{2}} \ldots j_{i_{r}}$ 


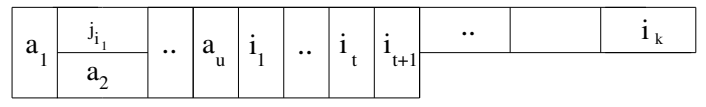

Figure 4

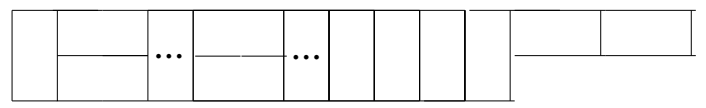

FiguRE 5

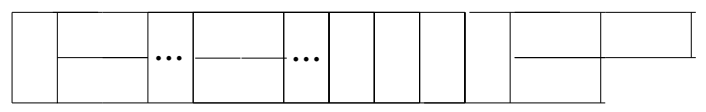

FiguRE 6

and $j_{\iota_{1}} j_{\iota_{2}} \ldots j_{\iota_{s}}$. Let us first assume that this shuffle is trivial in the sense that $z_{1}=j_{i_{1}} j_{i_{2}} \ldots j_{i_{r}} j_{\iota_{1}} j_{\iota_{2}} \ldots j_{\iota_{s}}$.

Let us consider the insertion of $z_{1}$ in $P$. If $j_{i_{1}}$ must be entered in the twoline part of $P$, say if $a_{1}<j_{i_{1}}<a_{2}$, the resulting domino will be as in Figure 4. that is, one vertical domino in $P$ becomes horizontal, and the first horizontal domino becomes vertical. If $j_{i_{2}}$ must also be entered in the two-line part of the tableau, the resulting tableau will look as in Figure 5 where once again a vertical domino becomes horizontal and a horizontal becomes vertical. Since the sequence $j_{i_{1}} j_{i_{2}} \ldots j_{i_{r}}$ is increasing this pattern is repeated until arriving at the elements that must be inserted in the one-line part of the tableau. These are inserted by bumping horizontal dominoes to the second line, giving tableaux of the form given in Figure 6. We next describe the insertion of the other elements of $z_{1}$, those from $j_{\iota_{1}} j_{\iota_{2}} \ldots j_{\iota_{s}}$. But this is much simpler, since the element to be inserted will always be bigger than those already inserted. It is therefore inserted as a horizontal domino at the end of the first line, without bumping.

This last description also shows that in general, when $z_{1}$ is a more complicated shuffle of $j_{i_{1}} j_{i_{2}} \ldots j_{i_{r}}$ and $j_{\iota_{1}} j_{\iota_{2}} \ldots j_{\iota_{s}}$, the insertion of the elements of $j_{\iota_{1}} j_{\iota_{2}} \ldots j_{\iota_{s}}$, does not influence the insertion of the elements of $j_{i_{1}} j_{i_{2}} \ldots j_{i_{r}}$. We have thus proved that the insertion of all elements of $z_{1}$ gives a two-line domino tableau.

Finally, we consider the insertion of the elements of $z_{2}$. But the elements of $z_{2}$ are all bigger than the elements of $P_{1}=P\left(\underline{i}_{1} \bar{a}_{1} \underline{i}_{2} \bar{a}_{2} \ldots \underline{i}_{u} \bar{a}_{u} z_{1}\right)$ and so they are inserted as horizontal dominoes at the end of $P_{1}$. To be precise, the resulting domino tableau is simply the concatenation of the lines of $P_{1}$ and $P\left(z_{2}\right)$. The theorem is proved.

In the remaining part of this section, we formulate a result which is a strong indication of the connection between the empty core case of the conjectures from BGIL and the representation theory of $b_{n}$, where $b_{n}$ is the blob algebra mentioned in the introduction.

Let $\operatorname{Par}_{\emptyset}(n)$ denote the set of integer partitions of degree $n$ with empty core and set $\operatorname{Par}_{\emptyset}:=\bigcup_{n \geq 0} \operatorname{Par}_{\emptyset}(n)$. Similarly, let $\operatorname{Bip}(n)$ denote the set of bipartitions $(\lambda, \mu)$ of total degree $n$ and set $\operatorname{Bip}:=\bigcup_{n \geq 0} \operatorname{Bip}(n)$. We denote by $S T_{\emptyset}(n), S T_{\emptyset}, S B T(n)$ and $S B T$ the set of standard (bi)tableaux with underlying shape in $\operatorname{Par}_{\emptyset}(n), \operatorname{Par}_{\emptyset}$, 
$\operatorname{Bip}(n)$ and Bip. For a $\lambda$ partition we denote by $\mathcal{Q}(\lambda)$ the two-quotient of $\lambda$; see for example [M] for a definition. Then $\mathcal{Q}(\lambda) \in \operatorname{Bip}(m)$ if $\lambda \in \operatorname{Par}_{\emptyset}(2 m)$ and $\mathcal{Q}$ induces a bijection

$$
\mathcal{Q}: \operatorname{Par}_{\emptyset} \rightarrow \text { Bip . }
$$

Following [BGIL we define a partial order on Bip by the rule

$$
(\lambda, \mu) \prec(\tau, \nu) \text { iff } \mathcal{Q}^{-1}(\lambda, \mu) \triangleleft \mathcal{Q}^{-1}(\tau, \nu)
$$

where $\triangleleft$ refers to the usual dominance order on partitions.

Let $\operatorname{Bip}_{1}(n)$ denote the set of one-line bipartitions of total degree $n$. An element of $\operatorname{Bip}_{1}(n)$ is of the form $(\lambda, \mu)=((a),(n-a))$ for some positive integer $a$ with $0 \leq a \leq n$. We shall use the shorthand notation $(a),(n-a)$ for $\operatorname{such}(\lambda, \mu)$ but reserve the notation $(a, n-a)$ for a conventional (two-line) partition. Set $\operatorname{Bip}_{1}:=\bigcup_{n \geq 0} \operatorname{Bip}_{1}(n)$.

Define $\Lambda_{n}:=\{-n,-n+2, \ldots, n-2, n\}$. Then there is a bijection:

$$
f: \operatorname{Bip}_{1}(n) \rightarrow \Lambda_{n}, \quad(a),(b) \mapsto a-b .
$$

Let $\prec$ (also) denote the order on $\Lambda_{n}$ induced by $f$, that is, for $\lambda, \mu \in \Lambda_{n}, \lambda \prec \mu$ iff $f^{-1}(\lambda) \prec f^{-1}(\mu)$.

Note that $\Lambda_{n}$ is the parameterizing set for the quasi-hereditary category $b_{n}$-mod of $b_{n}$-modules. The hereditary order is given by $\lambda<_{q h} \mu$ iff $|\lambda|>|\mu|$ for $\lambda, \mu \in \Lambda_{n}$. We now have the following result.

Theorem 2.11. a) $\operatorname{Bip}_{1}(n)$ is a coideal in Bip with respect to $\prec$.

b) The order $\prec$ on $\Lambda_{n}$ is a refinement of $<_{q h}$.

Proof. In CL a bijection $\overline{\mathcal{Q}}: S D T \rightarrow S B T$ is described. It induces $\mathcal{Q}: \operatorname{Par}_{\emptyset}(2 n) \rightarrow$ $\operatorname{Bip}(n)$ by taking shapes. One then checks the following formulas:

$$
\begin{array}{ll}
\mathcal{Q}^{-1}:(a),(b) \mapsto(2 a, 2 b) & \text { for } a \geq b, \\
\mathcal{Q}^{-1}:(a),(b) \mapsto(2 b-1,2 a+1) & \text { for } a<b .
\end{array}
$$

We deduce that $\mathcal{Q}^{-1}\left(\operatorname{Bip}_{1}(n)\right)$ consists of all partitions of $2 n$ of at most two lines and thus $\operatorname{Bip}_{1}(n)$ indeed is a coideal in Bip with respect to $\prec$ as claimed in a). In order to prove $b$ ) we note that the above formulas give

$$
(n),(\emptyset) \succ(\emptyset),(n) \succ(n-1),(1) \succ(1),(n-1) \succ(n-2),(2) \succ \ldots
$$

The last term is $\left(\frac{n}{2}\right),\left(\frac{n}{2}\right)$ or $\left(\frac{n-1}{2}\right),\left(\frac{n+1}{2}\right)$ depending on the parity of $n$. The statement of b) follows from this. In fact we see that the only difference between $\prec$ and $<_{q h}$ is that $-\lambda \prec \lambda$ if $\lambda \in \Lambda_{n}$ and $\lambda>0$, whereas they are noncomparable with respect to $<_{q h}$.

\section{Cell theory in $\mathcal{H}_{n}$}

The fundamental text on cell theory for Hecke algebras with unequal parameters is Lusztig's book $\mathrm{Lu2}$. Since here we are interested in the special $B_{n}$ case, we shall follow the notation used in BGIL]. Therefore, let $\Gamma$ be a finitely generated free Abelian group containing the elements $a, b$ and let $<$ be a total order on $\Gamma$, making it into an ordered group. We use exponential notation for the elements of $\Gamma$, writing $e^{g}$ for $g \in \Gamma$. Define $\mathbf{q}:=e^{a}$ and $\mathbf{Q}:=e^{b}$. Let $\mathcal{A}$ be the $\mathbb{Z}$-algebra 
$\mathcal{A}:=\mathbb{Z}[\Gamma]$. The Hecke algebra $\mathcal{H}_{n}=\mathcal{H}_{n}(\mathbf{Q}, \mathbf{q})$ of type $B$ is the $\mathcal{A}$-algebra generated by $T_{0}, T_{1}, \ldots, T_{n-1}$ subject to the relations

$$
\begin{array}{ll}
T_{i} T_{i-1} T_{i}=T_{i-1} T_{i} T_{i-1} & \text { for } i=2,3, \ldots, n-1, \\
T_{0} T_{1} T_{0} T_{1}=T_{1} T_{0} T_{1} T_{0}, & \\
T_{i} T_{j}=T_{j} T_{i} \text { for }|i-j|>1, & \\
\left(T_{i}-\mathbf{q}\right)\left(T_{i}+\mathbf{q}^{-1}\right)=0, & \left(T_{0}-\mathbf{Q}\right)\left(T_{0}+\mathbf{Q}^{-1}\right)=0 .
\end{array}
$$

The frequently used ground ring in the literature $\mathbb{Z}\left[\mathbf{Q}, \mathbf{Q}^{-1}, \mathbf{q}, \mathbf{q}^{-1}\right]$ is obtained as a special case of the above by setting $\Gamma:=\mathbb{Z} a \oplus \mathbb{Z} b$. The Hecke algebra defined over this ground ring is called the generic Hecke algebra.

Assume that $f: \Gamma \rightarrow \mathbb{C}^{\times}$is a group homomorphism. Then $f$ extends canonically to an algebra homomorphism $f: \mathcal{A} \rightarrow \mathbb{C}$ and we can define the specialized Hecke algebra $\mathcal{H}_{n, f}:=\mathcal{H}_{n} \otimes_{\mathcal{A}} \mathbb{C}$. For example, $f(g)=1, \forall g$ gives the group algebra $\mathcal{H}_{n, f}=\mathbb{C} W_{n}$.

Define elements $C_{i}$ of $\mathcal{H}_{n}$ by $C_{0}:=T_{0}-\mathbf{Q}$ and $C_{i}:=T_{i}-\mathbf{q}$ for $i=1,2, \ldots, n-1$. Let $J_{n}$ be the following ideal of $\mathcal{H}_{n}$,

$$
J_{n}:=\left\langle C_{1} C_{2} C_{1}-C_{1}, C_{1} C_{0} C_{1}-[2]_{\frac{\mathrm{Q}}{\mathrm{q}}} C_{1}\right\rangle
$$

where $[n]_{x}:=x^{n-1}+x^{n-3}+\ldots+x^{-n+3}+x^{-n+1}$ is the usual Gaussian integer. We then define the Temperley-Lieb algebra of type $B$ as

$$
T L B_{n}:=\mathcal{H}_{n} / J_{n} \text {. }
$$

In the case of the generic Hecke algebra, this definition already appears in GL1] where $T L B_{n}$ is also referred to as the blob algebra, but actually it differs slightly from the presentation of the blob algebra $b_{n}$ that is used, e.g., in [MR] and [RH]. Let us be more specific about the relationship.

Let $k$ be a field and assume that $q \in k^{\times}, q \neq 1,-1$ and $m \in \mathbb{Z}$. In $[\mathrm{RH}]$ and other references $b_{n}=b_{n}(q, m)$ is defined as the $k$-algebra on generators $U_{0}, U_{1}, U_{2} \ldots, U_{n-1}$ and relations

$$
\begin{aligned}
& U_{i} U_{i+1} U_{i}=U_{i} \text { for } i=1,2 \ldots, n-2, \\
& U_{i+1} U_{i} U_{i+1}=U_{i+1} \text { for } i=1,2 \ldots, n-2, \\
& U_{1} U_{0} U_{1}=[m-1] U_{1}, \\
& U_{i}^{2}=-[2] U_{i} \text { for } i=1,2 \ldots, n-1, \\
& U_{0}^{2}=-[m] U_{0}, \quad U_{i} U_{j}=U_{j} U_{i} \text { for }|i-j|>1,
\end{aligned}
$$

where $[a]=\frac{q^{a}-q^{-a}}{q-q^{-1}}$. The following lemma relates this to $T L B_{n}$.

Lemma 3.1. Suppose $k:=\mathbb{C}$. Assume $q \in \mathbb{C}^{\times} \backslash\{1,-1\}$ and set $Q:=i q^{m}$. Define $T L B_{n, q, Q}:=T L B_{n} \otimes_{\mathcal{A}} \mathbb{C}$ where $\mathbb{C}$ is made into an $\mathcal{A}$-algebra via $f: \Gamma \rightarrow \mathbb{C}^{\times}$such that $f(a)=q, f(b)=Q$. Then the rules

$$
C_{i} \mapsto U_{i}, \quad i=1,2, \ldots, n-1, \quad C_{0} \mapsto i\left(q-q^{-1}\right) U_{0}
$$

define an isomorphism $g: T L B_{n, q, Q} \rightarrow b_{n}(q, m)$.

Proof. It is shown in Proposition 2.1 of [CGM] that $T L B_{n}=\mathcal{H}_{n} / J_{n}$ is free over $\mathcal{A}=$ $\mathbb{Z}\left[\mathbf{Q}, \mathbf{Q}^{-1}, \mathbf{q}, \mathbf{q}^{-1}\right]$ and hence the proof is only a matter of checking the relations.

For $w \in W_{n}$ we define $T_{w}:=T_{i_{1}} T_{i_{2}} \ldots T_{i_{N}}$ where $w=s_{i_{1}} s_{i_{2}} \ldots s_{i_{N}}$ is a reduced expression. By the relations, $T_{w}$ is independent of the reduced expression. Moreover, $T_{w}$ is invertible since $T_{i}$ is invertible for all $i$; indeed we have

$$
T_{0}^{-1}=T_{0}-\mathbf{Q}+\mathbf{Q}^{-1}, \quad T_{i}^{-1}=T_{i}-\mathbf{q}+\mathbf{q}^{-1} \text { for } i=1,2, \ldots, n-1 .
$$


The bar involution $h \mapsto \bar{h}$ on $\mathcal{H}_{n}$ is the ring automorphism given by

$$
T_{w} \mapsto T_{w^{-1}}^{-1}, \quad \mathbf{q} \mapsto \mathbf{q}^{-1}, \quad \mathbf{Q} \mapsto \mathbf{Q}^{-1} .
$$

Recall now that $\Gamma$ is endowed with a total order $<$. Using it Lusztig introduces in [Lu1, Lu2] a Kazhdan-Lusztig type basis $C_{w}, w \in W_{n}$ for $\mathcal{H}_{n}$. It is uniquely defined by the conditions

$$
\overline{C_{w}}=C_{w}, \quad C_{w}-T_{w} \in \bigoplus_{w \in W_{n}} \mathcal{A}_{>0} T_{w}
$$

where $\mathcal{A}_{>0}:=\sum_{\gamma \in \Gamma, \gamma>0} \mathbb{C} e^{\gamma}$.

Associated with the basis $C_{w}$ there is a preorder $\leq_{L}$ on $W_{n}$, generated by $y \leq_{L} w$ if $C_{y}$ appears in the expansion of $C_{s_{i}} C_{y}$ in the $C_{w}$-basis. The associated equivalence relation is denoted $\sim_{L}$ and its classes are denoted left cells. Thus, $z \sim_{L} w$ if $z \leq w$ and $w \leq z$. Similarly, we define the preorders $\leq_{R}$ and $\leq_{L R}$ and the equivalence relations $\sim_{R}$ and $\sim_{L R}$. The associated classes are called right cells and two-sided cells.

We shall always assume that $a$ and $b$ are positive in $\Gamma$ and so by the equations (3.1) we get the following formulas:

$$
C_{s_{0}}=T_{0}-\mathbf{Q}, \quad C_{s_{i}}=T_{i}-\mathbf{q} \text { for } i=1,2, \ldots, n-1 .
$$

In other words, we have that $C_{s_{i}}=C_{i}$.

Assume that $b \notin\{a, 2 a, \ldots,(n-1) a\}$. Let $r \in \mathbb{N} \cup\{0\} \cup\{\infty\}$ be given by $r a<b<(r+1) a$ or $r:=\infty$ if $b>(n-1) a$. According to the conjectures in BGIL, the representation theory of $\mathcal{H}_{n}$ should only depend on $\Gamma, a$ and $b$ through $r$.

Let us consider the following $\mathcal{A}$-submodule of $\mathcal{H}_{n}$ :

$$
\mathcal{J}_{n}:=\operatorname{span}_{\mathcal{A}}\left\{C_{w} \mid w \notin W_{b}\right\} .
$$

The next theorem is the main result of this section. In order to formulate it, we recall that $c^{+}$of Conjecture A of BGIL is the statement that

$$
y \leq_{\mathcal{L} R} w \Longleftrightarrow \operatorname{Sh}(y) \leq S h(w) .
$$

Theorem 3.2. Assume that $r=0$ and assume that part $\mathrm{c}^{+}$of Conjecture $A$ of [BGIL] is valid for $r=0$. Then we have that $\mathcal{J}_{n}=J_{n}$.

Proof. Since $\mathrm{c}^{+}$is assumed to be true we have that $\leq_{\mathcal{L} R}$ is given by dominance order under domino insertion. Combining with Theorem 2.10 we get that $\mathcal{J}_{n}$ is an ideal in $\mathcal{H}_{n}$.

In order to show that $J_{n} \subset \mathcal{J}_{n}$ it is then enough to verify that the generators of $J_{n}$ belong to $\mathcal{J}_{n}$. Now we have

$$
\begin{aligned}
& C_{1} C_{2} C_{1}=\left(T_{1}-q\right)\left(T_{2}-q\right)\left(T_{1}-q\right) \\
& \quad=T_{1} T_{2} T_{1}-q T_{1} T_{2}-q T_{2} T_{1}+q^{2} T_{1}+q^{2} T_{2}-q^{3}+T_{1}-q
\end{aligned}
$$

and hence

$$
C_{s_{1} s_{2} s_{1}}=C_{1} C_{2} C_{1}-C_{1} .
$$

On the other hand, $P\left(s_{1} s_{2} s_{1}\right)$ has the form given in Figure 7 and so $C_{s_{1} s_{2} s_{1}} \in \mathcal{J}_{n}$. Similarly we have

$$
\begin{aligned}
& C_{1} C_{0} C_{1}=\left(T_{1}-q\right)\left(T_{0}-Q\right)\left(T_{1}-q\right) \\
& \quad=T_{1} T_{0} T_{1}-q T_{0} T_{1}+Q q^{-1} T_{1}-Q-q T_{1} T_{0}+q^{2} T_{0}+q Q T_{1}-q^{2} Q .
\end{aligned}
$$




\begin{tabular}{|l|l|l|l|l|l|}
\hline 1 & 4 & 5 &.. &.. & $\mathrm{n}$ \\
\hline 2 & \multicolumn{7}{|l}{} \\
\cline { 1 - 1 } 3 & \multicolumn{2}{|l}{} \\
\cline { 1 - 1 } &
\end{tabular}

Figure 7

\begin{tabular}{|c|c|c|c|c|c|c|}
\hline 1 & 3 & 4 &.. &.. & $\mathrm{n}$ \\
\hline 2 & \multicolumn{10}{|l}{} \\
\hline
\end{tabular}

Figure 8

But $-a+b<0$ and so $Q q^{-1} \notin \mathcal{A}_{>0}$ and we must subtract $[2]_{\frac{Q}{q}} C_{1}$ to get $C_{s_{1} s_{0} s_{1}}$. Hence

$$
C_{s_{1} s_{0} s_{1}}=C_{1} C_{0} C_{1}-[2]_{\frac{Q}{q}} C_{1} .
$$

But $P\left(s_{1} s_{0} s_{1}\right)$ is as in Figure 8 and so indeed $C_{s_{1} s_{0} s_{1}} \in \mathcal{J}_{n}$.

Let $K$ be the kernel of the projection map $\pi: \mathcal{H}_{n} / J_{n} \rightarrow \mathcal{H}_{n} / \mathcal{J}_{n}$. We need to show that $K=0$. Since $\pi$ is surjective, it is enough to prove that $\mathcal{H}_{n} / J_{n}$ and $\mathcal{H}_{n} / \mathcal{J}_{n}$ are free over $\mathcal{A}$ of the same rank.

As mentioned above, $T L B_{n}=\mathcal{H}_{n} / J_{n}$ was shown in CGM to be free over the ground ring $\mathbb{Z}\left[\mathbf{Q}, \mathbf{Q}^{-1}, \mathbf{q}, \mathbf{q}^{-1}\right]$. Its rank is given by the cardinality of the diagram basis and can also be read off from the Bratelli diagram for $T L B_{n}$. It is

$$
\operatorname{rank} \mathcal{H}_{n} / J_{n}=\sum_{i=0}^{n}\left(\begin{array}{c}
n \\
i
\end{array}\right)^{2}
$$

On the other hand, since $\left\{C_{w}\right\}$ is a basis of $\mathcal{H}_{n}$ we have that $\mathcal{H}_{n} / \mathcal{J}_{n}$ is free over $\mathcal{A}$ with rank

$$
\operatorname{rank} \mathcal{H}_{n} / \mathcal{J}_{n}=\left|W_{b}\right| \text {. }
$$

Recall the bijection $\overline{\mathcal{Q}}: S D T \rightarrow S B T$ from $\mathrm{CL}$. By the proof of Theorem 2.11. it restricts to a bijection between standard domino tableaux in $S T D(n)$ with less than two lines and one-line standard bitableaux with shape in $\operatorname{Bip}_{1}(n)$. The number of pairs of one-line bitableaux of shape $(i, n-i)$ is $\left(\begin{array}{c}n \\ i\end{array}\right)^{2}$ and so we conclude that $\operatorname{rank} \mathcal{H}_{n} / J_{n}=\operatorname{rank} \mathcal{H}_{n} / \mathcal{J}_{n}$, as needed.

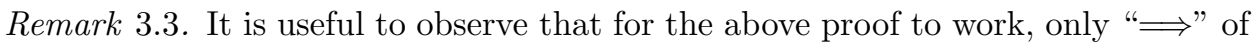
part $c^{+}$of Conjecture A from BGIL is actually needed.

Corollary 3.4. Assume that $\Gamma=\mathbb{Z}$ with the standard order and that $b=1$ and $a=2$. Then $\mathcal{J}_{n}=J_{n}$.

Proof. By Remark 4.1 of [BJ], which on the other hand relies on [Lu1, we get that $c^{+}$of Conjecture A of [BGIL] is valid under the assumptions. We then apply the theorem.

In order to apply the corollary, we shall from now on assume that $\Gamma:=\mathbb{Z}$ with the standard order, and that $b:=1, a=2$. Although this does not cover all of the $r=0$ case of BGIL we shall, somewhat misleadingly, refer to it that way. 
We need both versions of the blob algebra. Hence, in order for Lemma 3.1 and the corollary to work we impose the following conditions on $q, Q$ :

$$
q \text { is a primitive l'th root of unity, } l>2, Q:=i q^{m}, q=-q^{2 m} \text {. }
$$

Note that the conditions imply that $l$ is even. They will be satisfied, for example, if $l=2(2 m-1)$.

We choose from now on $q, Q, m, l$ satisfying (3.2). We use the notation $\mathcal{H}_{n, q, Q}$ for the specialized Hecke algebra $\mathcal{H}_{f}$ with respect to these choices. Similarly, we write $\mathcal{J}_{n, q, Q}$ for $\mathcal{J}_{f}$ and $J_{n, q, Q}$ for $J_{f}$.

Corollary 3.5. We have $T L B_{n, q, Q}=\mathcal{H}_{n, q, Q} / \mathcal{J}_{n, q, Q}=b_{n}(q, m)$.

Proof. This follows from the theorem and Lemma 3.1

\section{Representation theORY}

In this section we use the results of the previous sections to study the representation theory of $b_{n}$. Our main result is that the cell modules in the $r=0$ case are the standard modules for $b_{n}$.

Recall that $[2] \neq 0$ so that we can define $e=-\frac{1}{[2]} U_{n-1}$. This is an idempotent of $b_{n}$ and we have that $e b_{n} e \cong b_{n-2}$. Hence it gives rise to the localization functor

$$
F: b_{n}-\bmod \rightarrow b_{n-2}-\bmod , \quad M \mapsto e M .
$$

$F$ is exact, it has as left adjoint functor, the globalization functor $G$,

$$
G: b_{n-2}-\bmod \rightarrow b_{n}-\bmod , \quad M \mapsto b_{n} e \otimes_{e b_{n} e} M .
$$

Recall that $\Lambda_{n}:=\{-n,-n+2, \ldots, n-2, n\}$ is the parameterizing set for the quasihereditary category $b_{n}$-mod. Let $\Delta_{n}(\lambda) \in b_{n}$-mod denote the standard module associated with $\lambda \in \Lambda$. We have that

$$
\begin{aligned}
& F \Delta_{n}(\lambda) \cong \begin{cases}\Delta_{n-2}(\lambda) & \text { if } \lambda \in \Lambda_{n} \backslash\{ \pm n\} \\
0 & \text { otherwise }\end{cases} \\
& G \Delta_{n}(\lambda) \cong \begin{array}{l}
\Delta_{n+2}(\lambda),
\end{array}
\end{aligned}
$$

and $\Delta_{n}( \pm n) \cong L_{n}( \pm n)$ where $L_{n}(\lambda)$ is the simple module given by $\lambda$. This implies the universal property for $\Delta_{n}(\lambda)$ as the projective cover of $L_{n}(\lambda)$ in the truncated subcategory of $b_{n}$-mod consisting of modules with composition factors of the form $L_{n}(\mu)$ with $\mu \leq \lambda$.

Now, let $w_{n} \in W_{b}$ and denote by $\mathfrak{C}=\mathfrak{C}_{w_{n}} \subseteq W_{b}$ its left cell. Consider the following ideals of $\mathcal{H}_{n}$,

$$
\mathcal{I}_{\leq_{\mathfrak{c}} w_{n}}:=\operatorname{span}_{\mathbb{C}}\left\{C_{w} \mid w \leq_{L} w_{n}\right\}, \quad \mathcal{I}_{<\mathfrak{e} w_{n}}:=\operatorname{span}_{\mathbb{C}}\left\{C_{w} \mid w \leq_{L} w_{n}, w \notin \mathfrak{C}\right\}
$$

and define the cell module

$$
\mathcal{V}_{w_{n}}:=\mathcal{I}_{\leq_{\mathfrak{C}} w_{n}} / \mathcal{I}_{<\mathfrak{e} w_{n}}
$$

Since Conjecture A of BGIL is true in the $r=0$ case, we get by the results of the previous section that $\mathcal{V}_{w_{n}}$ is a $b_{n}$-module. A basis for $\mathcal{V}_{w_{n}}$ is given by the classes of $C_{w}$ for $w \in \mathfrak{C}$.

Recall from the previous sections that $W_{n}$ is realized as the subgroup of the symmetric group on the elements $I_{n}$ generated by $s_{0}:=(-1,1)$ and $s_{i}:=(i, i+$ $1)(-i,-i-1)$. Let us denote by $\iota$ the associated injection of groups $\iota: W_{n} \rightarrow S_{I_{n}}=$ $S_{2 n}$ :

$$
\iota\left(s_{0}\right)=(1,-1), \iota\left(s_{i}\right)=(i, i+1)(-i,-i+1) .
$$




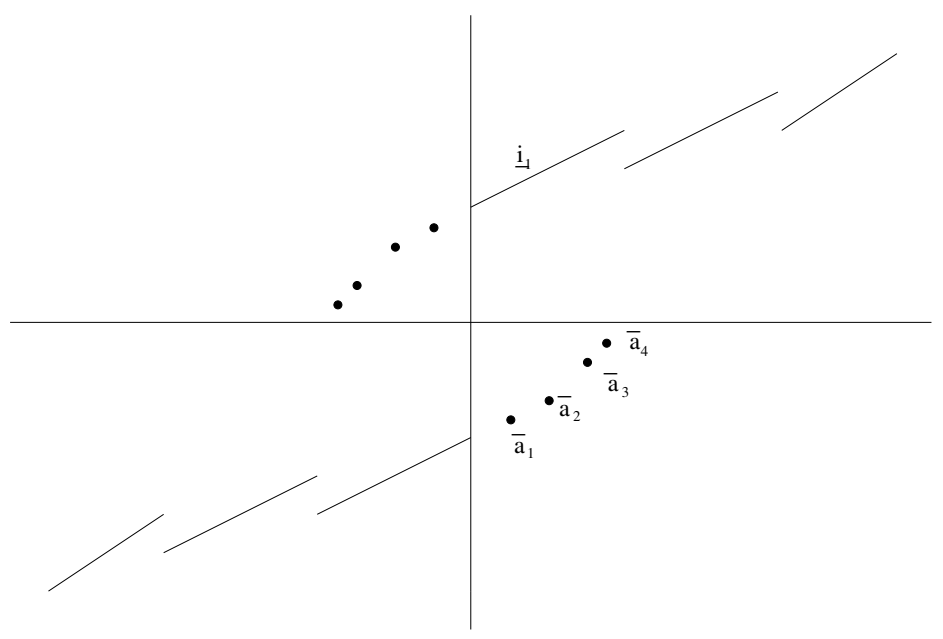

Figure 9

According to the last theorem of [Lu1] (on page 111), each left cell $\mathfrak{C}$ of $W_{n}$ is now of the form $\mathfrak{C}=\iota^{-1}(\widetilde{\mathfrak{C}})=\widetilde{\mathfrak{C}} \cap W_{n}$ where $\widetilde{\mathfrak{C}}$ is a left cell of $S_{I_{n}}$; this relies heavily on $r=0$.

By [KL, A1, G], the left cells on $S_{I_{n}}=S_{2 n}$ can be described using the usual Robinson-Schensted correspondence when we use the natural order on $I_{n}$, given by

$$
\bar{n}<\ldots<\overline{2}<\overline{1}<1<2<\ldots<n .
$$

We need the following lemma.

Lemma 4.1. Let $\mathfrak{C}$ be a left cell in $W_{n}$. Assume that $\mathfrak{C} \subset W_{b}$ and that $\mathfrak{C}=\widetilde{\mathfrak{C}} \cap W_{n}$ where $\widetilde{\mathfrak{C}}$ is a left cell of $S_{I_{n}}$. Then under the Robinson-Schensted bijection on $S_{I_{n}}$ with respect to the above order on $I_{n}, \widetilde{\mathfrak{C}}$ corresponds to a tableau in at most two lines.

Proof. Let $P^{\prime}$ and $Q^{\prime}$ denote the $P$ and $Q$-parts of the Robinson-Schensted correspondence on $S_{2 n}$. For $z, z_{1} \in \widetilde{\mathfrak{C}}$ we have $Q^{\prime}(z)=Q^{\prime}\left(z_{1}\right)$ and $P^{\prime}(z)$ and $Q^{\prime}(z)$ have the same shape. Assume now that $w \in W_{b}$ and write it in word form as $w=i_{1} i_{2} \ldots i_{n}$ with $i_{j} \in I_{n}$. We then have

$$
\iota(w)=\bar{w}^{o p} w
$$

where $\bar{w}^{o p}:=\bar{i}_{n} \bar{i}_{n-1} \ldots \bar{i}_{1}$ and so $P^{\prime}(\iota(w))=P^{\prime}\left(\bar{w}^{o p} w\right)$.

We now appeal to the description of $W_{b}$ given in Theorem 2.3. Using it, there are no decreasing subsequences of $\bar{w}^{o p} w$ of length three or more, and thus $P^{\prime}\left(\bar{w}^{o p} w\right)$ has at most two lines. Indeed, consider the graph of Figure 9. It represents $\bar{w}^{o p} w$ in the case where $\overline{a_{1}}, \ldots, \overline{a_{4}}$ are the only negative numbers in $w$, that is, $l=4$ in the notation of Theorem 2.3 . The restriction of the graph to the quadrants I and IV represents $w$ and the restriction of the graph to the quadrants I and II represents $w^{l}=w^{4}$ in the notation of Theorem [2.3. For simplicity, the straight lines of quadrants I and III represent sequences of increasing numbers, where $\underline{i}_{1}:=$ $i_{1} \ldots i_{k_{4}-1}$. From Theorem 2.3 we know that $w^{l}$ has no decreasing subsequences of length three or more, and that these are all positioned after $\underline{i}_{1}$ in the graph. Hence the discontinuity points of quadrant I will increase as indicated. We now conclude 
that $\bar{w}^{o p} w$ has no decreasing subsequences of length three or more, as claimed. The general case is treated the same way.

Lemma 4.2. a) Assume that $U_{n-1} C_{w_{n}} \neq 0$. Then there exists $w_{n-2} \in \mathfrak{C}_{w_{n}} \cap W_{n-2}$ and a scalar $a \in \mathbb{C} \backslash\{0\}$ such that $U_{n-1} C_{w_{n}}=a U_{n-1} C_{w_{n-2}}$.

b) Assume $U_{i} C_{w_{n}} \in \mathcal{V}_{w_{n}} \backslash\{0\}$ for some $i>0$. Then there exists $z \in \mathfrak{C}_{w_{n}}$ and a scalar $a \in \mathbb{C} \backslash\{0\}$ such that $U_{i} C_{w_{n}}=a C_{z}$.

c) Assume that $U_{n-1} \mathcal{V}_{w_{n}}=0$. Then $U_{i} \mathcal{V}_{w_{n}}=0$ for all $i>0$. Moreover, $\mathcal{V}_{w_{n}} \simeq \Delta_{n}( \pm n)$, especially $\operatorname{dim} \mathcal{V}_{w_{n}}=1$.

Proof. Take $w_{n} \in \mathfrak{C}_{w_{n}}=\mathfrak{C}$ and let $C_{w_{n}} \in \mathcal{H}_{n}$ be the associated Kazhdan-Lusztig element. Then we have

$$
U_{n-1} C_{w_{n}}=C_{s_{n-1}} C_{w_{n}}=\sum_{z \in W_{n}} N_{n-1, w_{n}, z} C_{z}
$$

where $N_{n-1, w_{n}, z}$ are the structure constants in $\mathcal{H}_{n}$ with respect to the $C$-basis. Let $\mathcal{H}_{2 n}$ be the Hecke algebra associated to $S_{2 n}$, with parameter $q$, and let us denote by $\widetilde{C}_{w}$ the usual one-parameter Kazhdan-Lusztig element for $w \in S_{2 n}$. If $w \in W_{n}$ we write $\widetilde{C}_{w}:=\widetilde{C}_{\iota(w)}$. Then we have

$$
\widetilde{C}_{s_{n-1}} \widetilde{C}_{w_{n}}=\sum_{z \in S_{2 n}} \widetilde{N}_{n-1, w_{n}, z} \widetilde{C}_{z}
$$

where $\widetilde{N}_{n-1, y, z}$ are the structure constants in $\mathcal{H}_{2 n}$ with respect to its $\widetilde{C}$-basis. Lusztig shows in this setting in [Lu1 that,

$$
\text { if } z \in W_{n} \text { and } N_{n-1, w_{n}, z} \neq 0 \text {, then } \widetilde{N}_{n-1, w_{n}, z} \neq 0 \text {. }
$$

Now we have

$$
\widetilde{C}_{s_{n-1}}=\left(T_{(n-1, n)}-q\right)\left(T_{(-n+1,-n)}-q\right)=U_{(n-1, n)} U_{(-n+1,-n)} .
$$

Reducing (4.2) modulo $\mathcal{I}_{<\mathcal{C} w_{n}}$ we get the corresponding equation in $\mathcal{V}_{w_{n}}$ :

$$
U_{n-1} C_{w_{n}}=\sum_{z \in \mathfrak{C}} N_{n-1, w_{n}, z} C_{z} \text { modulo } \mathcal{I}_{<\mathcal{C} w_{n}} .
$$

But $\mathfrak{C}=\widetilde{\mathfrak{C}} \cap W_{n}$ and so by (4.4) any $z$ occurring in this sum with $N_{n-1, w_{n}, z} \neq 0$ gives a nonzero $\widetilde{N}_{n-1, w_{n}, z}$ in

$$
\widetilde{C}_{s_{n-1}} \widetilde{C}_{w_{n}}=U_{(n-1, n)} U_{(-n+1,-n)} \widetilde{C}_{w_{n}}=\sum_{z \in S_{2 n}} \widetilde{N}_{n-1, w_{n}, z} \widetilde{C}_{z} \text { modulo } \mathfrak{I}
$$

where

$$
\mathfrak{I}:=\operatorname{span}_{\mathbb{C}}\left\{\widetilde{C}_{w} \mid w \in S_{2 n}, w \leq_{L} \widetilde{\mathfrak{C}}, w \notin \widetilde{\mathfrak{C}}\right\} .
$$

But using the previous lemma we may consider (4.6) as an equation in a cell module $\Delta_{2 n}(k)$ for the Temperley-Lieb algebra $T L_{2 n}$.

Let us now show a). We have $N_{n-1, w_{n}, z} \neq 0$ and so $\widetilde{N}_{n-1, w_{n}, z} \neq 0$. But by [FG] we know that $\widetilde{C}_{z}=U_{\iota(z)}$ modulo $\mathfrak{I}$, where as usual $U_{w}:=U_{i_{1}} \ldots U_{i_{r}}$ for $w=s_{i_{1}} \ldots s_{i_{r}}$. Using the diagram presentation of $\Delta_{2 n}(k)$ we now deduce that $\iota(z)=s_{(-n+1,-n)} z_{1} s_{(n-1, n)}$ where $z_{1} \in S_{I_{n-2}}$ and hence

$$
z_{1}=s_{(-n+1,-n)} \iota(z) s_{(n-1, n)}=\iota\left(z s_{n-1}\right) \in \iota\left(W_{n}\right) \cap S_{I_{n-2}}=\iota\left(W_{n-2}\right)
$$

and a) is proved. 
We then show b). For each $z$ with $N_{i, w_{n}, z} \neq 0$ we have by (4.4) that $\tilde{N}_{n-1, w_{n}, z} \neq 0$. But using $\left[\mathrm{FG}\right.$ ] once again, at most one $z$ can give $\widetilde{N}_{n-1, w_{n}, z} \neq 0$, proving b).

Let us then show c). By the previous sections, $\mathcal{V}_{w_{n}}$ is a module for $b_{n}$. Since $F \mathcal{V}_{w_{n}}=U_{n-1} \mathcal{V}_{w_{n}}=0$, it follows from the general representation theory of $b_{n}$ that

$$
\mathcal{V}_{w_{n}}=\Delta_{n}(n)^{k} \oplus \Delta_{n}(-n)^{l}
$$

for certain multiplicities $k, l$. Since $\mathcal{V}_{w_{n}}$ is a cell module, the products $C_{s_{i_{1}}} \ldots$ $C_{s_{i_{k}}} C_{w_{n}}$ generate $\mathcal{V}_{w_{n}}$. But by assumption only $C_{s_{0}}^{k} C_{w_{n}}=U_{0}^{k} C_{w_{n}} \in \mathcal{V}_{w_{n}}$ can be nonzero and since $U_{0}^{k}$ is a scalar multiple of $U_{0}$ we conclude that $k=1, l=0$ or $k=0, l=1$ and so $\operatorname{dim} \mathcal{V}_{w_{n}}=1$. The lemma is proved.

We are now in position to prove our main theorem.

Theorem 4.3. Assume that $q$ is a primitive l'th root of unity such that $q=-q^{2 m}$ and $Q:=i q^{m}$. Let $\mathfrak{C}=\mathfrak{C}_{w_{n}}$ be a left cell for $W_{n}$ and let $\mathcal{V}_{w_{n}}$ be the corresponding cell module. Then we have an isomorphism of $b_{n}$-modules

$$
\mathcal{V}_{w_{n}} \simeq \Delta_{n}(\lambda)
$$

where $\lambda=a-b$ for $\mathcal{Q}\left(S h\left(w_{n}\right)\right)=(a),(b)$.

Proof. Assume that $F \mathcal{V}_{w_{n}} \neq 0$ and consider the adjointness map $\varphi=\varphi_{w_{n}}: G \circ$ $F \mathcal{V}_{w_{n}} \rightarrow \mathcal{V}_{w_{n}}$. It is given concretely by multiplication

$$
\varphi: b_{n} e \otimes_{e b_{n} e} e \mathcal{V}_{w_{n}} \rightarrow \mathcal{V}_{w_{n}}, \quad U \otimes e v \mapsto U e v .
$$

Using b) of the previous lemma and the definition of left cells, we see that $\varphi$ is surjective.

We now prove that $\operatorname{ker} \varphi$ is zero. Recall from $\mathrm{MR}$ that $U_{i} U_{i+1} \ldots U_{n-1}$, where $i=0,1, \ldots, n-1$ generate $b_{n} e$ as an $e b_{n} e$-module. Using this and part a) of the previous lemma we can write any $k \in b_{n} e \otimes_{e b_{n} e} e \mathcal{V}_{w_{n}}$ in the form

$$
k=\sum_{i=0,1, \ldots, n-1} \sum_{w_{n-2} \in \mathfrak{C}_{w_{n}} \cap W_{n-2}} \lambda_{i, w_{n-2}} U_{i} U_{i+1} \ldots U_{n-1} \otimes_{e b_{n} e} U_{n-1} C_{w_{n-2}}
$$

where $\lambda_{i, w_{n-2}} \in \mathbb{C}$. Since $U_{n-1}$ and $C_{w_{n-2}}$ commute we have

$$
U_{n-1} C_{w_{n-2}}=-\frac{1}{[2]} U_{n-1} C_{w_{n-2}} U_{n-1}=-\frac{1}{[2]} e C_{w_{n-2}} e .
$$

Assume now that $k \in \operatorname{ker} \varphi$. We then get

$$
\begin{aligned}
k & =-\frac{1}{[2]} \sum_{i} \sum_{w_{n-2}} \lambda_{i, w_{n-2}} U_{i} U_{i+1} \ldots U_{n-1} \otimes_{e b_{n} e} e C_{w_{n-2}} e \\
& =-\frac{1}{[2]} \sum_{i} \sum_{w_{n-2}} \lambda_{i, w_{n-2}} U_{i} U_{i+1} \ldots U_{n-1} U_{n-1} C_{w_{n-2}} \otimes_{e b_{n} e} U_{n-1},
\end{aligned}
$$

which is zero since $k \in \operatorname{ker} \varphi$. This proves that $\varphi$ is an isomorphism.

Using a) of the previous lemma once again, we now deduce that

$$
F \mathcal{V}_{w_{n}} \simeq \mathcal{V}_{w_{n-2}} \text { for } w_{n}=w_{n-2} s_{n-1}, w_{n-2} \in \mathfrak{C}_{w_{n}} \cap W_{n-2}
$$

By Corollary 3.8 of BGIL, $w_{n-2}$ is independent of the choice of $w_{n}$. Under domino insertion, $S h\left(w_{n}\right)$ is obtained from $S h\left(w_{n-2}\right)$ by adding two horizontal dominoes, one at the end of each line. Hence, using the formulas for $\mathcal{Q}$ given in the proof of 
Theorem 2.11, we find that

$$
\mathcal{Q}\left(\operatorname{Sh}\left(w_{n-2}\right)\right)=(a-1),(b-1) \text { if } \mathcal{Q}\left(\operatorname{Sh}\left(w_{n}\right)\right)=(a),(b)
$$

and hence the difference is the same.

If $F \mathcal{V}_{w_{n}}=0$ we get by c) of the previous lemma that $\mathcal{V}_{w_{n}} \simeq \Delta_{n}( \pm n)$ and hence that $\operatorname{dim} \mathcal{V}_{w_{n}}=1$. But then the combinatorial description of left cells in terms of domino tableaux gives $w_{n}=1$ or $w_{n}=s_{0}$. For $w_{n}=1$ we have $\mathcal{Q}\left(\operatorname{Sh}\left(w_{n}\right)\right)=$ $(n),(\emptyset)$ whereas for $w_{n}=s_{0}$ we have $\mathcal{Q}\left(S h\left(w_{n}\right)\right)=(\emptyset),(n)$, compatible with the actions of $U_{0}$ in $\mathcal{V}_{w_{n}}$. The theorem is proved.

Remark 4.4. We think that the theorem is valid for more general choices of $q$ and $Q$ within $r=0$.

\section{THE FOCK SPACE}

In this section we give two applications of Theorem 4.3 that both rely on the Fock space approach to the representation theory of $\mathcal{H}_{n}$. The first gives a new proof of the main results of [MW] using Ariki's Theorem and the second settles the question of determining the Kleshchev bipartition that corresponds to the simple $b_{n}$-module $L_{n}(\lambda)$. To set this up we first need the following theorem.

Theorem 5.1. In the Grothendieck group of $b_{n}$-modules the equality $\Delta_{n}(\lambda)=$ $S_{n}(a, b)$ holds where $\lambda=a-b$ and $S_{n}(a, b)$ is the Dipper-James-Murphy Specht module for $\mathcal{H}_{n}$ corresponding to the bipartition $(a),(b)$.

Proof. This follows basically from Theorem 3 and Theorem 6 of [RH]. On the other hand, since $\underline{\mathrm{RH}}$ is based on a realization of $b_{n}$ as a quotient of the Ariki-Koike algebra $\mathcal{A} K_{n}\left(\lambda_{1}, \lambda_{2}, q\right)$ and a realization of $S_{n}(a, b)$ as a permutation module in the Ariki-Yamada-Terasoma tensor space for $\mathcal{A} K_{n}\left(\lambda_{1}, \lambda_{2}, q\right)$, we still give a few details on how to convert from one situation to the other.

Recall that $T L B_{n}=\mathcal{H}_{n} / J_{n}$ is free over $\mathcal{A}$ and so we have that

$$
T L B_{n, q, Q}=\mathcal{H}_{n, q, Q} / J_{n, q, Q} \text {. }
$$

By Lemma 3.1 we also know that $T L B_{n, q, Q}$ is isomorphic to the blob algebra $b_{n}(q, m)$, that on the other hand was realized in $[\mathrm{RH}]$ as $\mathcal{A} K_{n}\left(\lambda_{1}, \lambda_{2}, q\right) / G_{n}$ where $\lambda_{1}=\frac{q^{m}}{q-q^{-1}}, \lambda_{2}=\frac{q^{-m}}{q-q^{-1}}$ and $G_{n}$ is the ideal of $\mathcal{A} K_{n}\left(\lambda_{1}, \lambda_{2}, q\right)$ generated by $\left(X_{1} X_{2}-\right.$ $\left.\lambda_{1} \lambda_{2}\right)\left(g_{1}-q\right)$. The last realization requires the conditions $q^{4} \neq 1, \lambda_{1} \neq \lambda_{2}, \lambda_{1} \neq q^{2} \lambda_{2}$ and these conditions are imposed throughout in $[\mathrm{RH}]$.

Instead of converting directly between the Hecke algebra and the Ariki-Koike algebra setting we prefer to proceed as follows.

The Hecke algebra $\mathcal{H}_{n}$ is an Ariki-Koike algebra with parameters $\lambda_{1}=Q, \lambda_{2}=$ $-1 / Q$ and so we can develop the theory of $[\mathrm{RH}]$ entirely from the $\mathcal{H}_{n}$ point of view, once we have proved that $J_{n}$ acts trivially in the Ariki-Terasoma-Yamada tensor space $V^{\otimes n}$ for these choices of parameters when $\operatorname{dim} V=2$, corresponding to Theorem 1 of $[\mathrm{RH}]$.

Let us therefore detail how the analogue of Theorem 1 of $[\mathrm{RH}]$ is proved. Let $V$ be a complex vector space of dimension two and let $v_{1}, v_{2}$ be a basis. Let $R \in \operatorname{End}_{\mathbb{C}}(V \otimes V)$ be given by

$$
\begin{aligned}
& R\left(v_{i} \otimes v_{j}\right)=q v_{i} \otimes v_{j} \quad \text { if } i=j, \\
& R\left(v_{2} \otimes v_{1}\right)=v_{1} \otimes v_{2}, \\
& R\left(v_{1} \otimes v_{2}\right)=v_{2} \otimes v_{1}+\left(q-q^{-1}\right) v_{1} \otimes v_{2} .
\end{aligned}
$$


For $i=1,2, \ldots, n-1$, we let $T_{i} \in \mathcal{H}_{n}$ act in the tensor space $V^{\otimes n}$ by

$$
T_{i}:=I d^{\otimes i-1} \otimes R \otimes I d^{\otimes n-i-1} .
$$

For $v=v_{i_{1}} \otimes v_{i_{2}} \otimes \ldots \otimes v_{i_{k}} \otimes v_{i_{k+1}} \otimes \ldots \otimes v_{i_{n}}$, we define $S_{k} \in \operatorname{End}_{\mathbb{C}}\left(V^{\otimes n}\right)$ by

$$
S_{k}(v)= \begin{cases}q v_{i_{1}} \otimes v_{i_{2}} \otimes \ldots \otimes v_{i_{k}} \otimes v_{i_{k+1}} \otimes \ldots \otimes v_{i_{n}} & \text { if } i_{k}=i_{k+1} \\ v_{i_{1}} \otimes v_{i_{2}} \otimes \ldots \otimes v_{i_{k+1}} \otimes v_{i_{k}} \otimes \ldots \otimes v_{i_{n}} & \text { otherwise }\end{cases}
$$

and let $\varpi \in \operatorname{End}_{\mathbb{C}}\left(V^{\otimes n}\right)$ be given by

$$
\varpi\left(v_{i_{1}} \otimes v_{i_{2}} \otimes \ldots \otimes v_{i_{n}}\right):= \begin{cases}Q v & \text { if } i_{1}=1, \\ -Q^{-1} v & \text { if } i_{1}=2 .\end{cases}
$$

Setting

$$
T_{0}:=T_{1}^{-1} \ldots T_{n-2}^{-1} T_{n-1}^{-1} S_{n-1} S_{n-2} \ldots S_{1} \varpi
$$

it is then proved in [ATY] that the linear maps $T_{0}, T_{1}, \ldots, T_{n-1}$ define an action of $\mathcal{H}_{n}$ in $V^{\otimes n}$. Endowed with this action of $\mathcal{H}_{n}$, we call $V^{\otimes n}$ the Ariki-YamadaTerasoma tensor space.

Let us now show that the ideal $J_{n}$ is annihilated under this action. This is wellknown for the generator $C_{1} C_{2} C_{1}-C_{1}$ so we concentrate on $C_{1} C_{0} C_{1}-[2]_{\frac{Q}{q}} C_{1}$. Since $C_{1}$ acts semisimply in $\operatorname{span}\left\{v_{i} \otimes v_{j} \mid i, j=1,2\right\}$ with eigenvalue 0 of multiplicity three and eigenvalue - [2] of multiplicity one, it is enough to check the relation on vectors of the form $C_{1} v$ where $v=v_{2} \otimes v_{1} \otimes v_{i_{3}} \otimes \ldots \otimes v_{i_{n}}$ since $C_{1} v \neq 0$ for such $v$. But $C_{1} v=\left(v_{1} \otimes v_{2}-q v_{2} \otimes v_{1}\right) \otimes v_{i_{3}} \otimes \ldots \otimes v_{i_{n}}$ is an eigenvector for $C_{1}$ of eigenvalue $-[2]$ and hence it is enough to show that

$$
C_{1} C_{0}\left(v_{1} \otimes v_{2}-q v_{2} \otimes v_{1}\right) \otimes \bar{v}=[2]_{\frac{Q}{q}}\left(v_{1} \otimes v_{2}-q v_{2} \otimes v_{1}\right) \otimes \bar{v}
$$

where $\bar{v}=v_{i_{3}} \otimes \ldots \otimes v_{i_{n}}$. Let us consider the left-hand side of this equation. Using Lemma 1 of $[\mathrm{RH}]$, which is a reformulation of a result of [ATY], we find that

$$
C_{1} C_{0} q v_{2} \otimes v_{1} \otimes \bar{v}=q^{2}\left(Q+Q^{-1}\right) C_{1} v_{1} \otimes v_{2} \otimes \bar{v} .
$$

We then consider $C_{1} C_{0} v_{1} \otimes v_{2} \otimes \bar{v}$ which we rewrite as follows:

$$
\begin{array}{r}
C_{1} C_{0} v_{1} \otimes v_{2} \otimes \bar{v}=\left(T_{1}-q\right)\left(T_{0}-Q\right) v_{1} \otimes v_{2} \otimes \bar{v} \\
=\left(T_{1}-q\right) T_{0} v_{1} \otimes v_{2} \otimes \bar{v}-Q C_{1} v_{1} \otimes v_{2} \otimes \bar{v} .
\end{array}
$$

We consider here the first term $\left(T_{1}-q\right) T_{0} v_{1} \otimes v_{2} \otimes \bar{v}$ which we rewrite as follows:

$$
\begin{gathered}
\left(T_{1}-q\right) T_{0} v_{1} \otimes v_{2} \otimes \bar{v}=-q\left(T_{1}-q\right) T_{1} T_{0} T_{1} v_{2} \otimes v_{1} \otimes \bar{v} \\
=-q Q\left(T_{1}-q\right) v_{2} \otimes v_{1} \otimes \bar{v}=q^{2} Q C_{1} v_{1} \otimes v_{2} \otimes \bar{v}
\end{gathered}
$$

where we used for the second equality the argument given in the proof of Theorem 1 of $[\mathrm{RH}]$. Summing up, the LHS of (5.1) equals

$$
\left(-Q-q^{2} Q^{-1}\right) C_{1} v_{1} \otimes v_{2} \otimes \bar{v}
$$

which coincides with the RHS.

We can now develop the theory of $[\mathrm{RH}]$ from the Hecke algebra point of view. Especially for $\lambda \in \Lambda_{n}$ we define the permutation module

$$
M_{n}(\lambda):=\operatorname{span}_{\mathbb{C}}\left\{v_{i_{1}} \otimes v_{i_{2}} \otimes \ldots \otimes v_{i_{n}} \mid \#\left\{k: i_{k}=1\right\}-\#\left\{k: i_{k}=2\right\}=\lambda\right\}
$$

and get that $M_{n}(\lambda)$ satisfies the functorial properties for $F$ of (4.1).

Theorem 3 of $[\mathrm{RH}]$ is proved by induction. One checks that the inductive step works for all choices of the parameters satisfying $\lambda_{1} \neq \lambda_{2}$. But $\lambda_{1}=Q=i q^{m}$ and $\lambda_{2}=-Q^{-1}=i q^{-m}$ and so we have $\lambda_{1} / \lambda_{2}=q^{2 m}=-q \neq 1$, as needed. The 
induction basis is based on Lemma 3 of $[\mathrm{RH}$. The proof of that lemma works provided that $\lambda_{1}\left(q-q^{-1}\right) \neq q\left(\lambda_{1}-\lambda_{2}\right)$. But this is equivalent to $-q \neq q^{2}$, that is, $q \neq-1$, as needed.

Finally, the proof of Theorem 6 of $[\mathrm{RH}]$ claiming that $M_{n}(\lambda) \cong S_{n}(a, b)^{\circledast}$ is independent of the choices of the parameters and goes directly over. But in the Grothendieck group of $b_{n}$-modules, $S_{n}(a, b)$ is equal to its contragredient dual $S_{n}(a, b)^{\circledast}$, and so the proof of the theorem is finished.

Remark 5.2. In view of Theorem 4.3, an alternative proof might have been obtained using the results of section 4 of $[\mathrm{P} 1]$.

Remark 5.3. At this point we may remark that combining Theorem 4.3 with Lemma 2 of $[\mathrm{RH}]$, we get many examples of cells modules for different choices of $r$ that are not isomorphic. Indeed Lemma 2 of $[\mathrm{RH}]$ gives many examples of the adjointness map $G \circ F M_{n}(\lambda) \rightarrow M_{n}(\lambda)$ failing to be an isomorphism. Note that the condition in that lemma, that $q$ be an odd order root of unity, is not needed for showing that the adjointness map is not surjective - as is indeed mentioned in the proof of that lemma.

We now recall the Fock space approach to the representation theory of $\mathcal{H}_{n}$. Let $s=\left(s_{1}, s_{2}\right) \in \mathbb{Z}^{2}$ and let $\mathfrak{F}^{s}$ be the associated Fock space of level two. As a $\mathbb{C}(v)$-vector space it is given by

$$
\mathfrak{F}^{s}=\bigoplus_{\lambda \in \operatorname{Bip}} \mathbb{C}(v)|\lambda, s\rangle
$$

where $|\lambda, s\rangle$ is a symbol. Set $e:=l / 2$ where $l$ is the multiplicative order of $q$ as in (3.2). Let us briefly recall how $\mathfrak{F}^{s}$ becomes an integrable module for the quantum group $\mathcal{U}_{v}\left(\widehat{\mathfrak{s l}}_{e}\right)$, following the construction in [JMMO.

Since $\mathcal{U}_{v}\left(\widehat{\mathfrak{s l}}_{e}\right)$ is the $\mathbb{C}(v)$-algebra generated by $e_{i}, f_{i}, i=0,1, \ldots, e-1$ and $k_{h}, h \in \mathfrak{h}$ subject to certain well-known relations, it is enough to explain how these generators act in $\mathfrak{F}^{s}$. To any bipartition $\left(\lambda^{(1)}, \lambda^{(2)}\right)$ we associate its diagram

$$
\left\{(i, j, c) \mid c=1,2 \text { and } 1 \leq j \leq \lambda_{i}^{(c)}\right\} .
$$

For a node $\gamma=(i, j, c)$ of $\left(\lambda^{(1)}, \lambda^{(2)}\right)$ we define its $e$-residue by $\operatorname{res}_{e}(\gamma)=j-i+s_{c}$ mod $e$. We define a total order on the nodes of $\left(\lambda^{(1)}, \lambda^{(2)}\right)$ by $\gamma=(i, j, c)<\gamma^{\prime}=$ $\left(i^{\prime}, j^{\prime}, c^{\prime}\right)$ if $j-i+s_{c}<j^{\prime}-i^{\prime}+s_{c}^{\prime}$ or if $j-i+s_{c}=j^{\prime}-i^{\prime}+s_{c}^{\prime}$ and $c^{\prime}<c$ (notice this last inequality!). If $\lambda=\left(\lambda^{(1)}, \lambda^{(2)}\right)$ and $\mu=\left(\mu^{(1)}, \mu^{(2)}\right)$ are bipartitions such that $\lambda \subset \mu$ and $\gamma=\mu \backslash \lambda$ is an $i$-node we say that $\gamma$ is a removable $i$-node of $\mu$ and an addable $i$-node of $\lambda$ and we set

$$
\begin{aligned}
N_{i}^{>}(\lambda, \mu):= & \#\left\{\text { addable } i \text {-nodes } \gamma^{\prime} \text { of } \lambda \text { such that } \gamma^{\prime}>\gamma\right\} \\
& -\#\left\{\text { removable } i \text {-nodes } \gamma^{\prime} \text { of } \lambda \text { such that } \gamma^{\prime}>\gamma\right\}, \\
N_{i}^{<}(\lambda, \mu):= & \#\left\{\text { addable } i \text {-nodes } \gamma^{\prime} \text { of } \lambda \text { such that } \gamma^{\prime}<\gamma\right\} \\
& -\#\left\{\text { removable } i \text {-nodes } \gamma^{\prime} \text { of } \lambda \text { such that } \gamma^{\prime}<\gamma\right\} .
\end{aligned}
$$

The actions of $f_{i}, e_{i}$ on a basis vector of $\mathfrak{F}^{s}$ are now as follows:

$$
\begin{aligned}
& f_{i}|\lambda, s\rangle=\sum_{\mu, \operatorname{res}(\mu \backslash \lambda) \equiv i \bmod e} v^{N_{i}^{>}(\lambda, \mu)}|\mu, s\rangle \\
& e_{i}|\mu, s\rangle=\sum_{\lambda, \operatorname{res}(\mu \backslash \lambda) \equiv i \bmod e} v^{-N_{i}^{<}(\lambda, \mu)}|\lambda, s\rangle .
\end{aligned}
$$

There are similar formulas for the other generators. It is one of the important issues of the Fock space approach to the representation theory of $\mathcal{H}_{n}$ that $\mathfrak{F}^{s}$ with this action not only depends on the classes $s_{1} \bmod e$ and $s_{2} \bmod e$, but on $s$ itself. 
Let $\mathcal{U}_{v}\left(\widehat{\mathfrak{s l}}_{e}\right) \rightarrow \mathcal{U}_{v}\left(\widehat{\mathfrak{s l}}_{e}\right), u \mapsto \bar{u}$ be the bar involution given by

$$
\bar{v}:=v^{-1}, \quad \overline{f_{i}}:=f_{i}, \quad \overline{e_{i}}:=e_{i}, \quad \overline{k_{h}}:=k_{-h}
$$

and let $\mathfrak{F}^{s} \rightarrow \mathfrak{F}^{s}, x \mapsto \bar{x}$ be the bar involution of the Fock space constructed by Uglov in [U]. It satisfies $\overline{\emptyset, s\rangle}=\emptyset, s\rangle$ and is compatible with the bar involution on $\mathcal{U}_{v}\left(\widehat{\mathfrak{s l}}_{e}\right)$, that is $\overline{u x}=\bar{u} \bar{x}$ for $u \in \mathcal{U}_{v}\left(\widehat{\mathfrak{s l}}_{e}\right)$ and $x \in \mathfrak{F}^{s}$. By the results of [U] we get for $\lambda \in$ Bip a unique $G(\lambda, s) \in \mathfrak{F}^{s}$ such that

$$
\overline{G(\lambda, s)}=G(\lambda, s), \quad G(\lambda, s) \equiv|\lambda, s\rangle \quad \bmod v \mathbb{C}[v] \mathfrak{F}^{s} .
$$

For $\mu \in$ Bip write

$$
G(\mu, s)=\sum_{\lambda \in \operatorname{Bip}} d_{\lambda, \mu}^{s}(v)|\lambda, s\rangle .
$$

Set $\mathcal{M}[s]:=\mathcal{U}_{v}\left(\widehat{\mathfrak{s l}}_{e}\right)|\emptyset, s\rangle$. Then $\mathcal{M}[s]$ is an integrable module for $\mathcal{U}_{v}\left(\widehat{\mathfrak{s l}}_{e}\right)$ and so the crystal/canonical basis theory applies to it. In fact, there is a subset $\operatorname{Bip}_{e}^{s} \subset \operatorname{Bip}$ such that $G(\lambda, s)$ for $(\lambda, s) \in \operatorname{Bip}_{e}^{s}$ is the canonical basis/global crystal basis of $\mathcal{M}[s]$. Set $\operatorname{Bip}_{e}^{s}(n):=\operatorname{Bip}_{e}^{s} \cap \operatorname{Bip}(n)$. Assume that $m \equiv s_{1}-s_{2}$. Then by the deep theorem of Ariki in $\left[\mathrm{A}\right.$, we have that $\operatorname{Bip}_{e}^{s}(n)$ parameterizes the irreducible modules for $\mathcal{H}_{n}$ with corresponding decomposition numbers $d_{\lambda, \mu}^{s}(1)$.

The proof of our next theorem is essentially the same as the proof of Theorem 4.7 of [BJ], but notice that Theorem 4.7 of [BJ] requires the validity of the Conjectures $\mathrm{A}, \mathrm{B}$ and $\mathrm{B}^{\prime}$ of $\mathrm{BJ}$. As already mentioned, Conjecture A holds in the $r=0$ case whereas, as we shall see, we can replace Conjecture B by Theorem 4.3 and Conjecture $\mathrm{B}^{\prime}$ by our previous Theorem 5.1 .

Theorem 5.4. Let $m, l, e$ be as above and let $p$ be the largest integer such that $m+p e \leq 0$ and set $s:=(m+p e, 0)$. Then for $\mu \in \operatorname{Bip}_{e}^{s}(n)$ we have

$$
G(\mu, s)=|\mu, s\rangle+\sum_{\lambda \in \operatorname{Bip}(n), \lambda \prec \mu} d_{\lambda, \mu}^{s}(v)|\lambda, s\rangle .
$$

Moreover, identifying $\tau=\left(t_{1}, t_{2}\right) \in \operatorname{Bip}_{1}(n)$ with $f(\tau)=t_{1}-t_{2} \in \Lambda_{n}$ we have for $\lambda, \mu \in \operatorname{Bip}_{1}$ that

$$
\left[\Delta_{n}(\lambda), L_{n}(\mu)\right]=d_{\lambda, \mu}^{s}(1) .
$$

Proof. By the choice of $s$ we have formula (5.2) as in the proof of Theorem 4.7 of BJ]. Notice now that $m+p e \neq 0$. Thus we have that $\lambda$ and $\mu$ of (5.3) belong to $\operatorname{Bip}_{e}^{s}(n)$. These bipartitions were studied in FLOTW and are called FLOTW bipartitions in $\mathrm{Ja}, \mathrm{BJ}$, and other references.

Now take $\nu=\left(n_{1}, n_{2}\right) \in \operatorname{Bip}_{1}(n)$ corresponding to $\nu \in \Lambda_{n}$. According to Ariki's Theorem there exists $\mu \in \operatorname{Bip}_{e}^{s}(n)$ such that the decomposition number $d_{\lambda, \nu}:=$ $\left[S_{n}(\lambda), L_{n}(\nu)\right]$ satisfies

$$
\left[\Delta_{n}(\lambda), L_{n}(\nu)\right]=d_{\lambda, \nu}=d_{\lambda, \mu}^{s}(1)
$$

for all $\lambda \in \operatorname{Bip}(n)$ where we used Theorem 5.1 for the first equality. Setting $\lambda=\nu$ we get that $\nu \preceq \mu$ and setting $\lambda=\mu$ we get that $\mu \preceq \nu$. Hence $\mu=\nu$ and the theorem is proved.

The next step is now to calculate the numbers $d_{\lambda, \mu}^{s}(1)$ for $\lambda, \mu \in \operatorname{Bip}(n)$. Uglov's proof of the existence of $G(\lambda, s)$ is not straightforward, but still constructive. Notice that his algorithm has been simplified by Yvonne in $[\mathrm{Y}$, and that Fayers, [Fa], as 
well as Jacon, Ja1, have found combinatorial generalizations of the LLT algorithm to higher levels.

On the other hand, since here we only focus on bipartitions in $\operatorname{Bip}_{1}(n)$, the properties of $G(\lambda, s)$ already mentioned are sufficient to calculate $G(\lambda, s)$ and hence $d_{\lambda, \mu}^{s}(1)$.

Indeed, set $m_{-}:=-(m+(p+1) e)$ and recall from $\mathrm{MW}$ that the choices of $e$ and $m$ determine an alcove geometry in $\mathbb{R}$ with zero dimensional walls in the integral points $\mathcal{M}:=\left\{m_{-}+k e \mid k \in \mathbb{Z}\right\}$ and fundamental alcove $A_{0}$ being the one that contains 0 . The associated Weyl group $\mathcal{W}$ is infinite dihedral, generated by $s_{+}$ and $s_{-}$where $s_{+}\left(s_{-}\right)$is the reflection in the right (left) wall of the fundamental alcove. Set $\Lambda_{n}^{r e g}:=\Lambda_{n} \backslash \mathcal{M}$ and for $\lambda \in \Lambda_{n}^{r e g}$ write $A_{\lambda}$ for the alcove containing $\lambda$. For $\lambda \in \Lambda_{n}^{r e g}$ we define $w_{\lambda} \in \mathcal{W}$ by the condition $w_{\lambda} A_{0}=A_{\lambda}$. Thus $w_{\lambda}<w_{\mu}$ in the Bruhat-Chevalley order implies $\lambda>\mu$ in the quasi-hereditary order. We can now formulate the next theorem. The second part of it was proved in [MW] using completely different methods.

Theorem 5.5. Let $\lambda, \mu \in \Lambda_{n}^{r e g}$. Then we have

$$
\begin{gathered}
d_{\lambda, \mu}^{s}(v)=\left\{\begin{array}{cc}
v^{l\left(w_{\lambda}\right)-l\left(w_{\mu}\right)}, & \text { if } w_{\lambda} \leq w_{\mu}, \\
0 & \text { otherwise }
\end{array}\right. \\
{\left[\Delta_{n}(\lambda), L_{n}(\mu)\right]=\left\{\begin{array}{cc}
1 & \text { if } w_{\lambda} \leq w_{\mu}, \\
0 & \text { otherwise. }
\end{array}\right.}
\end{gathered}
$$

Proof. Following [MW] we enumerate the elements of $\mathcal{W}$ as,

$$
w_{i}= \begin{cases}1 & \text { if } i=0 \\ s_{-} s_{+} s_{i} \ldots(-i \text { terms }) & \text { if } i<0 \\ s_{+} s_{-} s_{+} \ldots(i \text { terms }) & \text { if } i>0\end{cases}
$$

and define $A_{i}:=w_{i} A_{0}$. Then, $A_{i}$ is the alcove at distance $i$ from $A_{0}$, positioned to the right if $i$ is positive and to the left if $i$ is negative.

Write $s_{1}:=m+$ pe such that $s=\left(s_{1}, 0\right)$. Set furthermore $m_{+}:=m_{-}+e$. Then the fundamental alcove is limited by $m_{-}$and $m_{+}$. Assume now that $\lambda=\left(k_{1}, k_{2}\right)$ belongs to $A_{i} \cap \Lambda_{n}^{r e g}$ with $i \geq 0$. Let $r_{1}, r_{2} \in\{0,1, \ldots, e-1\}$ be the residues modulo $e$ of $k_{1}+s_{1}, k_{2}$.

We now act with elements of the form $f_{r_{1}+j} \ldots f_{r_{1}+1} f_{r_{1}}$ in $|\lambda, s\rangle$ and consider the images in $\mathfrak{F}^{s, \geq 2}:=\mathfrak{F}^{s} / I^{\geq 2}$ where $I^{\geq 2}:=\operatorname{span}\left\{|\nu, s\rangle \mid \nu \notin \operatorname{Bip}_{1}\right\}$. These images move towards the right wall of $A_{i}$. The wall will be reached when $r_{1}+j=r_{2} \bmod e$ and the image will be $|\mu, s\rangle$ where $\mu=\left(k_{1}+r_{1}-r_{2}, k_{2}\right)$, i.e. with $v$ power equal to $v^{0}$ since $k \geq 0$. Notice here that the wall $m_{+}$of $A_{0}$ corresponds exactly to the second case in the definition of the order relation on the nodes.

In the formalism of translation functors, as exposed for example in [S], the process just described corresponds to translation upwards on the wall.

Acting with $f_{r_{1}}$ in $|\mu, s\rangle$ and considering the images in $\mathfrak{F}^{s, \geq 2}:=\mathfrak{F}^{s} / I^{\geq 2}$ the result is

$$
\left|\mu^{u p}, s\right\rangle+v\left|\mu^{\text {down }}, s\right\rangle
$$

where $\mu^{u p}=\left(k_{1}+1, k_{2}+r_{1}-r_{2}\right)$ and $\mu^{\text {down }}=\left(k_{1}, k_{2}+r_{1}-r_{2}+1\right)$ and once again we get correspondence with the translation functor formalism. 


\section{TABLE 1}

\begin{tabular}{|c|c|}
\hline \multicolumn{2}{|c|}{$e=3, m=2, s=(-1,0)$} \\
\hline $\operatorname{Bip}_{1}(10)$ & $\mathrm{KBip}_{1}(10)$ \\
\hline$(10),(\emptyset)$ & $(10),(\emptyset)$ \\
\hline$(9),(1)$ & $(9),(1)$ \\
\hline$(8),(2)$ & $((8,1),(1))$ \\
\hline$(7),(3)$ & $((7,2),(1))$ \\
\hline$(6),(4)$ & $((6,3),(1))$ \\
\hline$(5),(5)$ & $((5,4),(1))$ \\
\hline$(4),(6)$ & $((4,2),(4))$ \\
\hline$(3),(7)$ & $((5,1),(4))$ \\
\hline$(2),(8)$ & $(6),(4)$ \\
\hline$(1),(9)$ & $(7),(3)$ \\
\hline$(\emptyset),(10)$ & $(8),(2)$ \\
\hline
\end{tabular}

\begin{tabular}{|c|c|}
\hline \multicolumn{2}{|c|}{$e=5, m=3, s=(-2,0)$} \\
\hline $\operatorname{Bip}_{1}(10)$ & $\mathrm{KBip}_{1}(10)$ \\
\hline$(10),(\emptyset)$ & $(10),(\emptyset)$ \\
\hline$(9),(1)$ & $(9),(1)$ \\
\hline$(8),(2)$ & $(8),(2)$ \\
\hline$(7),(3)$ & $((7,1),(2))$ \\
\hline$(6),(4)$ & $((6,2),(2))$ \\
\hline$(5),(5)$ & $((5,3),(2))$ \\
\hline$(4),(6)$ & $((4,4),(2))$ \\
\hline$(3),(7)$ & $(4),(6)$ \\
\hline$(2),(8)$ & $(5),(5)$ \\
\hline$(1),(9)$ & $(6),(4)$ \\
\hline$(\emptyset),(10)$ & $(7),(3)$ \\
\hline
\end{tabular}

Similarly, we go through the other cases and find that translation upwards through the wall behaves as above whereas translation downwards through the wall $|\mu, s\rangle$ is given by

$$
v^{-1}\left|\mu^{u p}, s\right\rangle+\left|\mu^{\text {down }}, s\right\rangle
$$

where $\mu^{u p}$ and $\mu^{\text {down }}$ are chosen analogously to the first case.

Using these rules, together with (5.2) and Theorem 2.11 it is now straightforward to calculate $G(\lambda, s)$ modulo $I^{\geq 2}$ for $\lambda \in \operatorname{Bip}_{1}$ to obtain formula (5.4). Finally, formula (5.5) then follows from the previous theorem.

Let us finish by mentioning one more application of our results. The (negative) Kleshchev bipartitions (see [Ja], [BJ] and references therein), are by definition the bipartitions that belong to $\operatorname{Bip}_{e}^{s}$ where $s=(d+r e, 0)$ is chosen such that $d+r e>$ $n-1-e$, this is the so-called (negative) asymptotic case. The Kleshchev bipartitions parameterize the simple modules for $\mathcal{H}_{n}$ when we use the dual Specht modules to parameterize, that is when we use the $M_{n}(\lambda)$ 's.

The question raised in $[\mathrm{RH}]$ of determining the Kleshchev bipartition $\lambda=\left(l_{1}, l_{2}\right)$ that corresponds to the simple $b_{n}$-module with parameter $\tau=\left(t_{1}, t_{2}\right)$ can now be solved by applying Kashiwara's operators to the crystal graphs of the Fock spaces. Consider as an example $e=3, m=2$. Then $s=(-1,0)$. In the crystal graph of $\mathcal{M}(-1,0)$ we have

$$
\tilde{f}_{0} \tilde{f}_{1} \tilde{f}_{0} \tilde{f}_{2} \tilde{f}_{2} \tilde{f}_{1} \tilde{f}_{1} \tilde{f}_{0} \tilde{f}_{0} \tilde{f}_{2}(\emptyset, \emptyset)=(6,4)
$$

whereas the same sequence of crystal operators sends $(\emptyset, \emptyset)$ to $((6,3),(1))$ in $\mathcal{M}(11,0)$.

Jacon has constructed in [Ja] an algorithm for converting between such crystal graphs. Tables 1 and 2 have been calculated using an implementation of his algorithm in the GAP system. They convert between the bipartitions in $\operatorname{Bip}_{1}(10)$ and the corresponding Kleshchev bipartitions, that we denote $\operatorname{KBip}_{1}(10)$.

It can be seen that the correspondence between $\operatorname{Bip}_{1}(10)$ and $\operatorname{KBip}_{1}(10)$ works as the identity in the top $m$ lines of all of these tables. This is a consequence of the following last result. 
TABLE 2

\begin{tabular}{|c|c|}
\hline \multicolumn{2}{|c|}{$e=7, m=4, s=(-3,0)$} \\
\hline $\operatorname{Bip}_{1}(10)$ & $\mathrm{KBip}_{1}(10)$ \\
\hline$(10),(\emptyset)$ & $(10),(\emptyset)$ \\
\hline$(9),(1)$ & $(9),(1)$ \\
\hline$(8),(2)$ & $(8),(2)$ \\
\hline$(7),(3)$ & $(7),(3)$ \\
\hline$(6),(4)$ & $((6,1),(3))$ \\
\hline$(5),(5)$ & $((5,2),(3))$ \\
\hline$(4),(6)$ & $((4,3),(3))$ \\
\hline$(3),(7)$ & $(3),(7)$ \\
\hline$(2),(8)$ & $(4),(6)$ \\
\hline$(1),(9)$ & $(5),(5)$ \\
\hline$(\emptyset),(10)$ & $(6),(4)$ \\
\hline
\end{tabular}

\begin{tabular}{|c|c|}
\hline \multicolumn{2}{|c|}{$e=9, m=5, s=(-4,0)$} \\
\hline $\operatorname{Bip}_{1}(10)$ & $\mathrm{KBip}_{1}(10)$ \\
\hline$(10),(\emptyset)$ & $(10),(\emptyset)$ \\
\hline$(9),(1)$ & $(9),(1)$ \\
\hline$(8),(2)$ & $(8),(2)$ \\
\hline$(7),(3)$ & $(7),(3)$ \\
\hline$(6),(4)$ & $(6),(4)$ \\
\hline$(5),(5)$ & $((5,1),(4))$ \\
\hline$(4),(6)$ & $((4,2),(4))$ \\
\hline$(3),(7)$ & $((3,3),(4))$ \\
\hline$(2),(8)$ & $(3),(7)$ \\
\hline$(1),(9)$ & $(4),(6)$ \\
\hline$(\emptyset),(10)$ & $(5),(5)$ \\
\hline
\end{tabular}

Proposition 5.6. For $n_{2}=0,1, \ldots, m-1$ we have that $\Delta_{n}(\lambda) \cong S_{n}\left(n_{1}, n_{2}\right)^{\circledast}$ where $\lambda:=n_{1}-n_{2}$. In particular, for these values of $n_{2}$ the irreducible $b_{n}$-module $L_{n}(\lambda)$ is isomorphic to the unique irreducible quotient of $S_{n}\left(n_{1}, n_{2}\right)^{\circledast}$.

Proof. We already saw that $S_{n}\left(n_{1}, n_{2}\right)^{\circledast} \cong M_{n}\left(n_{1}, n_{2}\right)$. Let $N$ be the minimum of $n_{1}$ and $n_{2}$. Then we get by the categorical properties (4.1), that

$$
\Delta_{n}(\lambda) \cong G^{\circ N} \circ F^{\circ N} \Delta_{n}(\lambda)
$$

where $F^{\circ N}:=F \circ \ldots \circ F$ and $G^{\circ N}:=G \circ \ldots \circ G$ are the $N$-fold compositions of the functors $F$ and $G$. On the other hand, using Lemma 2 of $[\mathrm{RH}$, we find for the chosen values of $n_{2}$, a similar description of $M_{n}\left(n_{1}, n_{2}\right)$ as follows:

$$
M_{n}\left(n_{1}, n_{2}\right) \cong G^{\circ N} \circ F^{\circ N} M_{n}\left(n_{1}, n_{2}\right) .
$$

Notice that the conditions of that lemma on $l$ to be odd and $n_{2} \neq m \bmod l$ can be replaced by $n_{2} \neq m \bmod e$, as can easily be seen from the proof of the lemma. We now use Theorem 2 and Lemma 3 of loc. cit. together with (4.1) to deduce that

$$
F^{\circ N} \Delta_{n}(\lambda) \cong F^{\circ N} M_{n}\left(n_{1}, n_{2}\right),
$$

and the proposition follows by applying $G^{\circ N}$ to this isomorphism.

Unfortunately, in general we do not have a nonrecursive description of the elements of $\operatorname{KBip}_{1}(n)$.

\section{REFERENCES}

[A] S. Ariki, On the decomposition numbers of the Hecke algebra of $G(m, 1, n)$, J. Math. Kyoto Univ. 36 (1996), 789-808. MR.1443748 (98h:20012)

[A1] S. Ariki, Robinson-Schensted correspondence and left cells, Advanced studies in Pure Mathematics 28, 2000, Combinatorial methods in Representation Theory pp. 1-20. MR.1855588 (2002i:20003)

[ATY] S. Ariki, T. Terasoma, H. Yamada, Schur-Weyl reciprocity for the Hecke algebra of $\mathbb{Z} / r \mathbb{Z}$ 乙 $\mathfrak{S}_{n}$, J. Algebra 178 (1995), 374-390. MR1359891 (96k:20010)

[BV] D. Barbasch, D. Vogan, Primitive ideals and orbital integrals on complex classical groups, Math. Ann. 259 (1982), pp. 153-199. MR656661(83m:22026)

[BB] A. Björner, F. Brenti, Combinatorics of Coxeter groups. Graduate Texts in Mathematics, 231. Springer, New York, 2005. MR2133266 (2006d:05001) 
[B] C. Bonnafé, Two-sided cells in type B (asymptotic case), Journal of Algebra 304, (2006), 216-236. MR2255826 (2007j:20003)

[BGIL] C. Bonnafé, M. Geck, L. Iancu and T. Lam, On domino insertion and Kazhdan-Lusztig cells in type $B_{n}$. In: Representation theory of algebraic groups and quantum groups (Nagoya, 2006; eds. A. Gyoja et al.), pp. 33-54, Progress in Math. 284, Birkhäuser, 2010. MR2761947 (2011k:20004)

[BI] C. Bonnafé, L. Iancu, Left cells in type $B_{n}$ with unequal parameters, Representation Theory 7, (2003), 587-609. MR2017068(2004j:20007)

[BJ] C. Bonnafé, N. Jacon, Cellular structures on Hecke algebras of type B, J. Algebra, 321, Issue 11, 2009, 3089-3111. MR2510041 (2010f:20005)

[CGM] A. G. Cox, J. Graham, P. P. Martin, The blob algebra in positive characteristic, J. Algebra 266, No. 2, 584-635 (2003) MR1995129 (2004f:20012)

[DJM] R. Dipper, G. James, E. Murphy, Hecke algebras of type $B_{n}$ at roots of unity, Proc. London. Math. Soc., 70 (1995), 505-528. MR1317512 (96b:20004)

[CL] C. Carré, B. Leclerc, Splitting the square of a Schur function into its symmetric and antisymmetric parts. J. Algebraic Combin. 4 (1995), no. 3, 201-231. MR.1331743 (97b:05165)

[Fa] M. Fayers, An LLT-type algorithm for computing higher-level canonical bases, J. Pure Appl. Algebra 214 (2010), 2186-98. MR2660908 (2011g:17026)

[FG] C. K. Fan, R. M. Green, Monomials and Temperley-Lieb Algebras, J. of Algebra 190 Issue 2, (1997), 498-517. MR1441960 (98a:20037)

[FLOTW] O. Foda, B. Leclerc, M. Okado, J.-Y. Thibon, T. Welsh, Branching functions of $A_{n-1}^{(1)}$ and Jantzen-Seitz problem for Ariki-Koike algebras, Advances in Mathematics 141 (1999), 322-365. MR 1671762 (2000f:17036)

[G] M. Geck, Kazhdan-Lusztig cells and the Murphy basis, Proceedings of the London Mathematical Society 93 (2006), pp. 635-665. MR2266962 (2008f:20012)

[Ga] D. Garfinkle, On the classification of primitive ideals for complex classical Lie algebras II, Compositio Math. 81 (1992), pp. 307-336. MR1149172 (93g:17018)

[GL] J. Graham, G.I. Lehrer, Cellular algebras, Inventiones Math. 123 (1996), 1-34. MR1376244(97h:20016)

[GL1] J. Graham, G.I. Lehrer, Diagram algebras, Hecke algebras and decomposition numbers at roots of unity, Ann. Scient. Éc. Norm. Sup., 4e série, 36, 2003, pp. 479-524. MR2013924 (2004k:20007)

[JMMO] M. Jimbo, K. Misra, T. Miwa and M. Okado, Combinatorics of representations of $U_{q}\left(\widehat{\mathfrak{s l}}_{n}\right)$ at $q=0$, Comm. Math. Phys. 136 (1991), 543-566. MR.1099695 (93a:17015)

[KL] D. A. Kazhdan and G. Lusztig, Representations of Coxeter groups and Hecke algebras, Invent. Math. 53 (1979), 165-184. MR.560412 (81j:20066)

[La] T. Lam, Growth diagrams, domino insertion, and sign-imbalance, J. Comb. Theor. Ser. A. 107 (2004), 87-115. MR2063955 (2005g:05153)

[Lu1] G. Lusztig, Left cells in Weyl groups, Lie Group Representations, I (eds. R. L. R. Herb and J. Rosenberg), Lecture Notes in Mathematics 1024 (Springer, Berlin, 1983), pp. 99-111. MR727851 (85f:20035)

[Lu2] G. Lusztig, Hecke algebras with unequal parameters, CRM Monographs Ser. 18, Amer. Math. Soc., Providence, RI, 2003. MR.1974442 (2004k:20011)

[Ja] N. Jacon, Crystal graphs of irreducible $\mathcal{U}_{v}\left(\mathfrak{s} l_{e}\right)$-modules of level two and Uglov bipartitions, J. Algebraic Combin. 27 (2008), no. 2, p. 143-162. MR2375488 (2009c:17017)

[Ja1] N. Jacon, An algorithm for the computation of the decomposition matrices for ArikiKoike algebras, J. Algebra 292 (2005), 100-9. MR2166797 (2006g:20010)

[M] I. Macdonald, Symmetric Functions and Hall Polynomials, Oxford University Press, 1995. MR1354144 (96h:05207)

[MR] P. P. Martin, S. Ryom-Hansen, Virtual Algebraic Lie Theory, Tilting modules and Ringel duality for blob algebras, Proc. London Math. Soc. 89 (2004), 655-675. MR2107010 (2005i:20007)

[MS] P. P. Martin, H. Saleur, The blob algebra and the periodic Temperley-Lieb algebra, Lett. Math. Phys. (1994) 30, 189-206. MR1267001 (95e:81105)

[MW] P. P. Martin, D. Woodcock, On the structure of the blob Algebra, J. Algebra 225, (2000), 957-988. MR1741573 (2000k:16015) 
[P] T. Pietraho, Knuth Relations for the Hyperoctahedral Groups, J. of Algebraic Combin, 29 (4), (2009), 509-535. MR2506719 (2010c:20050)

[P1] T. Pietraho, Module Structure of Cells in Unequal Parameter Hecke Algebras, Nagoya Mathematical Journal, 198 (2010), 23-45. MR2666576 (2011h:20007)

[RH] S. Ryom-Hansen, The Ariki-Terasoma-Yamada tensor space and the blob algebra, J. of Algebra 324 (2010), 2658-2675. MR2725194 (2012b:20008)

[U] D. Uglov, Canonical bases of higher level q-deformed Fock spaces and Kazhdan-Lusztig polynomials, Kashiwara, Masaki (ed.) et al., Boston: Birkhäuser. Prog. Math. 191 (2000), 249-299. MR1768086 (2001k:17030)

[Sch97] Martin Schonert et al. GAP - Groups, Algorithms, and Programming - version 3 release 4 patchlevel 4 " Lehrstuhl Dür Mathematik, Rheinisch Westfälische Technische Hochschule, Aachen, Germany, 1997.

[Sa] B. E. Sagan, The Symmetric Group: Representations, Combinatorial Algorithms, and Symmetric Functions, 2nd edition, Springer-Verlag, New York, 2001. MR,1824028 (2001m:05261)

[S] W. Soergel, Kazhdan-Lusztig-Polynome und eine Kombinatorik für Kipp-Moduln, Represent. Theory 1 (1997), 37-68. MR 1445511 (99d:17023)

[T] M. Taskin, Plactic relations for $r$-domino tableaux, Electron. J. Combin., volume 19, issue 1, (2012), P38. MR2880669

[vL] M. van Leeuwen, The Robinson-Schensted and Schutzenberger algorithms, an elementary approach, The Foata Festscrhift, Electron. J. Combin. volume 3, issue 2 (1996), P15. MR1392500 (97e:05200)

[Y] X. Yvonne, An algorithm for computing the canonical bases of higher-level q-deformed Fock spaces, J. Algebra 309 (2007), 760-785. MR2303205 (2009b:17042)

Instituto de Matemática y Física, Universidad de Talca, Chile

E-mail address: steen@inst-mat.utalca.cl 